\title{
Pure Strategy Nash Equilibria of Large Finite-Player Games and their Relationship to Non-Atomic Games*
}

\author{
Guilherme Carmona $^{\dagger} \quad$ Konrad Podczeck ${ }^{\ddagger}$ \\ University of Surrey Universität Wien
}

January 6, 2020

\begin{abstract}
We consider Nash equilibria of large anonymous games (i.e., each player's payoff depends on his choice and the distribution of the choices made by others). We show that pure strategy Nash equilibria exist in all sufficiently large finiteplayer games with finite action spaces and for generic distributions of players' payoff functions. We also show that equilibrium distributions of non-atomic games are asymptotically implementable in terms of Nash equilibria of large finite-player games. Extensions of these results to games with general compact metric action spaces are provided.
\end{abstract}

Journal of Economic Literature Classification Numbers: C72.

*We wish to thank Michael Greinecker, Mário Páscoa, the editor (Marciano Siniscalchi), two anonymous referees and seminar participants at the Universität Innsbruck, Games 2016 (Maastricht) and EWGET 2017 (Salamanca) for helpful comments. Any remaining errors are, of course, ours.

${ }^{\dagger}$ Address: University of Surrey, School of Economics, AD Building, Guildford, GU2 7XH, UK; email: g.carmona@surrey.ac.uk.

$\ddagger$ Address: Institut für Volkswirtschaftslehre, Universität Wien, Oskar-Morgenstern-Platz 1, A1090 Wien, Austria; email: konrad.podczeck@univie.ac.at 
Keywords: Large games, pure strategy, Nash equilibrium, asymptotic implementation, generic property.

\section{Introduction}

Addressing economic problems by models of strategic interaction with an infinite number of negligible individuals (non-atomic games for short) is now common and much progress has been witnessed in the theory of large games over the last years. ${ }^{1}$ However, some fundamental questions remain unanswered:

1. Non-atomic games have equilibria in pure strategies (e.g. Schmeidler (1973) and Mas-Colell (1984)); to what extent does this property hold in games with a large finite number of players?

2. Are equilibria of non-atomic games limits of equilibria of large finite games?

As it has been discussed since the early days of the theory of non-atomic models in economics (see Section 2 for a literature review), these issues are fundamental to assert to what extent are non-atomic games a good idealization of games that, in reality, have a large but finite set of players. In this paper we provide answers to these questions.

We address these questions in the context of games with anonymous players, in the sense that each player's payoff function depends only on his own action and on the distribution of actions chosen by the other players, with a compact set of possible payoff functions; this latter condition means that, in such non-atomic games, there is a bound on the diversity of players' payoffs functions. ${ }^{2}$ We first focus on games with finite action spaces.

\footnotetext{
${ }^{1}$ Recent papers on large games include Gradwohl and Reingold (2010), Khan, Rath, Sun, and Yu (2013), Carmona and Podczeck (2014), Deb and Kalai (2015), Khan, Rath, Yu, and Zhang (2017), Yang (2017) and Kalai and Shmaya (2018). See the references therein for further recent work and Khan and Sun (2002) for a survey of earlier work.

${ }^{2}$ Kalai (2004) refers to the games we consider as semi-anonymous; the need for the qualifier "semi" arises in his paper because, unlike us, he allows for incomplete information which, in turn, allows that a player's identity be encoded in his type. We note that elsewhere (e.g. Khan and Sun
} 
Regarding the first question above, we note that not all sufficiently large games have a pure strategy Nash equilibrium as we show by an example. Thus, a generic existence result is the best one can hope for. And, indeed, we show that all sufficiently large games whose distribution of payoff functions is sufficiently close to a generic distribution of payoff functions of a non-atomic game have a pure strategy Nash equilibrium. Thus, the existence of equilibria in pure strategies, which is a general property of non-atomic games, holds generically in games with a large finite number of players. The notion of a generic set we use, which is that of an open and dense set, gives a reasonable sense to the assertion that our generic existence result applies to all but "exceptional" games.

We answer the second question above affirmatively. Indeed, we show that any equilibrium (distribution) of any non-atomic game (with a compact set of possible payoff functions) is asymptotically implementable in the following sense: There exists a sequence of finite player games converging to the non-atomic game and a corresponding sequence of pure strategy Nash equilibria whose distributions converge to the equilibrium distribution of the non-atomic game. ${ }^{3}$ This holds for general nonatomic games, not just the generic ones. This bears some surprise: It is well known that there is often an "explosion" of Nash equilibria in the limit, which suggested that limits of pure strategy Nash equilibria in sequences of finite-player games converging to a given non-atomic game could form a proper subset of the equilibrium set of the limit non-atomic game. As our result shows, this intuition is incorrect.

Our results go beyond existing results in important aspects. Results analogous to ours have been obtained for pure strategy approximate equilibria, among others, by Rashid (1983), Khan and Sun (1999), Kalai (2004), Carmona and Podczeck (2009), Carmona and Podczeck (2012b) and Deb and Kalai (2015) (who have established (1999)) the notion of "anonymous game" is reserved for non-atomic games specified by distributions of players' characteristics.

${ }^{3}$ This notion of "asymptotic implementation" is more specific than that in Khan and Sun (1996), where "asymptotic implementation" signifies, in the sense of nonstandard analysis, a "transfer" of any results for games with a hyperfinite Loeb spaces of players to games with a finite number of players (see Loeb and Wolff (2015, p. 355)). 
the existence of pure strategy approximate equilibria in sufficiently large finite-player games), and by Housman (1988) and Carmona and Podczeck (2012a) (who has established the asymptotic implementation of equilibria of non-atomic in terms of approximate equilibria of large finite-player games). These results left open the questions of existence of pure strategy Nash equilibria, which we now settled affirmatively in the case of generic games, and asymptotic implementation in terms of pure strategy Nash equilibria, which we have now settled affirmatively in the general case.

The importance of obtaining results for Nash equilibria of large finite-player games and not just for approximate equilibria arises because the latter are not always appealing. To see this, consider a game where each player can choose either the number 0 or 1 , and each player's payoff is obtained by multiplying his action with the average choice of the entire population (i.e. including his own choice). If this game is played by $n$ players, then the pure strategy profile where each player chooses 0 is an $\varepsilon$-equilibrium with $\varepsilon=1 / n$ : Indeed, no player can change the average choice and, thus, his payoff by more than $1 / n$ by changing his own action. In contrast, there is a unique pure strategy Nash equilibrium, namely the strategy in which all players choose 1; this is, in fact, a strictly dominant strategy.

In contrast, this issue does not arise in our two main results and, consequently, they provide a stronger sense under which non-atomic games are, indeed, a proper idealization of games with a large but finite number of players.

We also consider the case where players' actions can vary continuously, namely by allowing for action spaces that can be any compact metric space. Our asymptotic implementation result extends to this more general case. We also provide an extension of our generic existence result for large finite-player games to the case of general compact metric actions spaces. Specifically, this result establishes the generic existence of mixed strategy Nash equilibria that are nearly pure in the sense that each player's mixed strategy is concentrated on a set of "small" diameter. In other words, it is virtually impossible to distinguish such a nearly pure mixed strategy from a pure strategy. We emphasize that these strategies are still Nash equilibria, i.e. best-replies to themselves. 
In our companion paper, Carmona and Podczeck (2019), we show that, under differentiability assumptions in the case where players' actions can vary continuously, mixed strategies can be completely dispensed with in our results. Here, however, players' action sets can be finite or have isolated points and, therefore, no differentiability assumptions are made and no differentiable methods are used. In fact, apart from the requirement that the set of players' payoff functions be compact, our description of large games is completely standard.

The generality of our framework means that our results apply to many economic problems. To illustrate, we present two economic applications to which our results apply: The allocation, to a large number of individuals and through an anonymous mechanism, of indivisible objects and schools. The formalization of both of these applications is quite general, meaning, in particular, that we do not restrict attention to a particular mechanism or to a restricted set of players' preferences. To illustrate that our results extend to more restrictive formalizations, we also consider the allocation of indivisible objects through a specific mechanism, the first-price auction, and when players' preferences are restricted to depend only on the probability and value of obtaining the object, and on the bid submitted. It is interesting to note that while a general treatment of discontinuous large games is outside the scope of this paper, our results extend to a setting, such as the one in this latter application, where players' payoff functions are discontinuous.

The paper is organized as follows. A brief review of the literature is provided in Section 2. Section 3 contains some motivating examples. Our model is presented in Section 4. Our results on the generic existence of pure strategy Nash equilibria in large finite-player games are in Section 5. In Section 6 we consider the asymptotic implementation of equilibrium distributions of non-atomic games. The extension of our results to the case of games with a compact action space is in Section 7. Section 8 contains our economic applications and Section 9 provides some concluding remarks. The proofs of our results are in the Appendix. 


\section{Literature review}

The use in economics of models featuring a continuum of agents started with Aumann (1964). Such models are convenient from a mathematical point of view, and a way of expressing the idea, central to many economic applications, that each individual alone cannot influence aggregate statistics such as the price level or, more generally, the distributions of actions taken by all participants in a game.

But models with a continuum of agents are idealizations of reality which, of course, features a finite number of people. Despite Aumann's (1964) convincing arguments in favor of non-atomic models, many were unconvinced and dismissed them as being unrealistic; ${ }^{4}$ motivated or independently of the resulting debate, many found it natural enough to ask what is the relationship between economic models featuring a large but finite number of player and their non-atomic counterparts: For instance, Hildenbrand (1970), Brown and Robinson (1972), Hildenbrand (1974), Mas-Colell (1985), Rashid (1987) and Anderson (1991) in general equilibrium theory, and Rashid (1983), Green (1984), Housman (1988), Rashid (1992), Khan and Sun (1996), Khan and Sun (1999), Khan and Sun (2002), Kalai (2004) and Carmona and Podczeck (2009) in game theory. We will focus on the latter strand of the literature in the remainder of this section.

One way of relating large finite-player and non-atomic games is to ask whether the limit of a converging sequence of equilibria of finite-player games is an equilibrium in the limit non-atomic game. This was shown to be the case by Green (1984); see also Qiao and Yu (2014) for a recent result along these lines.

Asymptotic implementation, which is one of the foci of our paper, concerns the other direction in the relationship between large finite-player and non-atomic games. Namely, it starts with an equilibrium of the non-atomic game and asks whether sufficiently large finite-player games close to it have (approximate) equilibria close to the starting equilibrium. Bearing some repetition from the introduction, results related to ours have been obtained by Housman (1988) and Carmona and Podczeck

\footnotetext{
${ }^{4}$ For instance, Koopmans (1974) refers to the work of Aumann (1964) and others as "fanciful" and Georgescu-Roegen (1979) as "one of the most incriminating corpora delicti of empty mathematization". See Khan (2014) for an analysis of Georgescu-Roegen's (1979) criticism.
} 
(2012a), where the asymptotic implementation of equilibria of non-atomic in terms of approximate equilibria of large finite-player games has been demonstrated. Our contribution consists in dropping the "approximate" qualifier in those results.

A related way of relating large finite-player and non-atomic games is to ask whether a property that is known to hold for non-atomic games, such as the existence of pure strategy Nash equilibria, also holds for all sufficiently large finite-player games. Rashid (1983), Khan and Sun (1999), Kalai (2004), Carmona and Podczeck (2009), Carmona and Podczeck (2012b) and Deb and Kalai (2015) have established the existence of pure strategy approximate equilibria in sufficiently large finite-player games; as above, our contribution consists in dropping the "approximate" qualifier in those results in the generic case.

In several papers, including the recent ones of Khan, Rath, Sun, and Yu (2013), Qiao and Yu (2014) and Khan, Rath, Yu, and Zhang (2017), non-atomic games are described using an explicit set of players and a measurable function assigning payoff functions to them. While we describe non-atomic games only by a distribution over players' payoff functions, our results can be easily translated to the former setting: Indeed, the distribution of the payoff-assigning function is a non-atomic game in the sense of our paper and the distribution of the payoff-assigning function and of a Nash equilibrium (i.e. a measurable function from players to actions) is an equilibrium distribution.

\section{Motivating examples}

Consider a large population of individuals who face a coordination problem. The optimal choice of any individual depends on the choice of all others through their influence on how popular each of the two options is.

Example 1. To make the example specific, assume that each individual in the population has to choose one of two options, 0 or 1 ; thus, there is a common action set $A=\{0,1\}$. The relative frequencies with which the options are chosen are described by the vector $\pi=\left(\pi_{0}, \pi_{1}\right)$ in the unit simplex $\Delta$ of $\mathbb{R}^{2} ; \pi$ is referred to as the action 
distribution. Half of the population has preferences that are maximized when the action chosen matches the most frequent action. The payoff function of each of these players is denoted by $u_{c}$ and is defined by setting, for each $a \in A$ and $\pi \in \Delta$,

$$
u_{c}(a, \pi)= \begin{cases}\pi_{0}-\frac{1}{2} & \text { if } a=0 \\ 0 & \text { if } a=1\end{cases}
$$

The remaining half of the population preferences that are maximized when the action chosen matches the least frequent action. The payoff function of each of these players is denoted by $u_{d}$ and is defined by setting, for each $a \in A$ and $\pi \in \Delta$,

$$
u_{d}(a, \pi)= \begin{cases}\frac{1}{2}-\pi_{0} & \text { if } a=0 \\ 0 & \text { if } a=1 .\end{cases}
$$

Example 1 is specified without being explicit about the population of players. This is done so that we can address the question of whether or not a pure strategy Nash equilibrium exists when the above game is played by a sufficiently large population. To this end, suppose that the above coordination game is played by a population of $n$ players, where $n \in \mathbb{N}, n \geq 4$ and even. Specifically, each of these $n$ players chooses one of the two options in $A$, half of them have payoff function $u_{c}$ and the remaining half have payoff function $u_{d}$. The action distribution that is relevant for each player $i$ is the average distribution induced by the choices of the others

$$
\sum_{j \neq i} \frac{1_{a_{j}}}{n-1}
$$

where $a_{j}$ is the action chosen by player $j$ and $1_{a_{j}} \in \Delta$ equals $(1,0)$ if $a_{j}=0$ and $(0,1)$ if $a_{j}=1$. Thus, the relative frequency with which action 0 is chosen by players other than $i$ is

$$
e_{i}:=\sum_{j \neq i} \frac{1_{a_{j}}(0)}{n-1}=\frac{\#\left(\left\{j \neq i: a_{j}=0\right\}\right)}{n-1} .
$$

Example 1 shows that not all sufficiently large games have pure strategy Nash equilibria. Specifically, the following property fails: There exists $N \in \mathbb{N}$ such that for all $n \geq N$, the $n$-player version of the game in Example 1 has a pure strategy Nash equilibrium. 
To see this, let $n \geq 4$ and even be given and suppose that $a^{*}=\left(a_{1}^{*}, \ldots, a_{n}^{*}\right)$ is a pure strategy Nash equilibrium. Let $C$ (resp. D) be the set of player with payoff function $u_{c}\left(\right.$ resp. $\left.u_{d}\right)$. Consider $i, i^{\prime} \in C$ and suppose $a_{i}^{*}=0$ and $a_{i^{\prime}}^{*}=1$. Then, by optimal choice of actions, $e_{i} \geq 1 / 2 \geq e_{i^{\prime}}$. On the other hand, calculating frequencies, we see that $a_{i}^{*}=0$ and $a_{i^{\prime}}^{*}=1$ together imply that

$$
e_{i}=\frac{\#\left(\left\{j \in\{1, \ldots, n\}: a_{j}^{*}=0\right\}\right)-1}{n-1}<\frac{\#\left(\left\{j \in\{1, \ldots, n\}: a_{j}^{*}=0\right\}\right)}{n-1}=e_{i^{\prime}}
$$

and from this contradiction it follows that all members of $C$ must choose the same action, say 0 . But then, for all members $i$ of $D, e_{i} \geq \frac{n / 2}{n-1}>1 / 2$ because $\#(C)=n / 2$, so they all must play 1 , by optimal choice of actions. This, however, means that, for all members $i$ of $C, e_{i}=\frac{n / 2-1}{n-1}<1 / 2$ because $\#(C)=\#(D)=n / 2$, so their optimal actions are also equal to 1 , and this contradiction shows that no pure strategy Nash equilibrium exists.

Our results will show that the above example is non-generic. This conclusion is illustrated easily in the following example.

Example 2. Consider a set of games parameterized by $x \in[0,1]$. Specifically, for each $x \in[0,1]$, define a game as in Example 1 except that $u_{d}(1, \pi)=-\frac{x}{2}$ for each $\pi \in \Delta$. In particular, note that the game in Example 1 corresponds to $x=0$.

Consider a sequence of $n$-player games such that, for each $n \in \mathbb{N}$, each player has payoff function $u_{c}$ or $u_{d}$, and the fraction of players having payoff function $u_{c}$ converges to $1 / 2$. We now show that for each $x \in[0,1] \backslash\{0\}$, there is an $N \in \mathbb{N}$ such that each game in the sequence has a pure strategy Nash equilibrium whenever $n \geq N$; hence, this conclusion holds in an open and dense subset of the parameter space. $^{5}$

To see this, let $n \in \mathbb{N}$ be given and $C_{n}$ (resp. $D_{n}$ ) be the set of players with payoff function $u_{c}\left(\right.$ resp. $\left.u_{d}\right)$. Define $a_{n}^{*}=\left(a_{n, 1}^{*}, \ldots, a_{n, n}^{*}\right)$ as follows: $a_{n, i}^{*}=0$ for all players

\footnotetext{
${ }^{5}$ The need to have a large number of players can be seen by considering the matching pennies game: This is a two-player game such that all 2-player games in an open neighborhood of itself have a unique equilibrium in mixed strategies.
} 
$i \in C_{n}$ and for $\eta_{n}$ players in $D_{n} ; a_{n, i}^{*}=1$ for the remaining players. Letting $\nu_{n}$ be the fraction of players having payoff function $u_{c}$, pick $\eta_{n}$ such that

$$
\begin{aligned}
& \frac{1}{2}<\frac{n \nu_{n}+\eta_{n}-1}{n-1}, \\
& \frac{n \nu_{n}+\eta_{n}-1}{n-1} \leq \frac{1+x}{2}, \text { and } \\
& \frac{n \nu_{n}+\eta_{n}}{n-1} \geq \frac{1+x}{2} .
\end{aligned}
$$

These inequalities imply that all players are best-replying in $a_{n}^{*}$ and, thus, that $a_{n}^{*}$ is a Nash equilibrium: The first applies to players in $C_{n}$, the second to those players in $D_{n}$ who play 0 and the third to those players in $D_{n}$ who play 1 . The second and third inequality hold if and only if $\eta_{n} \in\left[\gamma_{n}, \gamma_{n}+1\right]$, where $\gamma_{n}=\frac{(n-1)(1+x)}{2}-n \nu_{n}$; as there is an integer in $\left[\gamma_{n}, \gamma_{n}+1\right]$, let $\eta_{n}$ be one such integer. Since $\frac{\eta_{n}}{n} \geq \frac{\gamma_{n}}{n} \rightarrow \frac{x}{2}>0, \eta_{n}>0$ for all $n$ sufficiently large. Also, for each $n, \eta_{n} \leq \gamma_{n}+1 \leq n-1-n \nu_{n}+1=n-n \nu_{n}=$ $n\left(1-\nu_{n}\right)$. Moreover, the first inequality holds if and only if $\eta_{n}>\frac{n-1}{2}-n \nu_{n}+1$; as $\lim _{n}\left(\frac{n-1}{2 n}-\nu_{n}+\frac{1}{n}\right)=0<\lim _{n} \frac{\gamma_{n}}{n} \leq \lim _{n} \frac{\eta_{n}}{n}$, it follows that the first inequality is also satisfied for all $n$ sufficiently large.

Example 3. Consider the game of Example 1 played by a continuum of players.

In this case, the population is not explicitly described, but rather a distributional view is adopted in that only the fraction of those players having a given payoff function and choosing a given action is specified. An equilibrium distribution of this game is a probability measure $\tau=\left(\tau_{\left(u_{c}, 0\right)}, \tau_{\left(u_{c}, 1\right)}, \tau_{\left(u_{d}, 0\right)}, \tau_{\left(u_{d}, 1\right)}\right)$ on $\left\{u_{c}, u_{d}\right\} \times\{0,1\}$ (with the interpretation that $\tau_{(u, a)}$ is the fraction of players with payoff function $u$ and who choose action $a$ ) such that, for each $u \in\left\{u_{c}, u_{d}\right\}, \tau_{(u, 0)}+\tau_{(u, 1)}=1 / 2$ and, for each $(u, a) \in\left\{u_{c}, u_{d}\right\} \times\{0,1\}$ with $\tau_{(u, a)}>0, u\left(a, \tau_{A}\right) \geq u\left(a^{\prime}, \tau_{A}\right)$ for all $a^{\prime} \in A$ (where $\left.\tau_{A}=\left(\tau_{\left(u_{c}, 0\right)}+\tau_{\left(u_{d}, 0\right)}, \tau_{\left(u_{c}, 1\right)}+\tau_{\left(u_{d}, 1\right)}\right) \in \Delta\right)$. It is easy to see that the set of equilibrium distributions consists of $\tau$ such that $\tau_{(u, 0)}+\tau_{(u, 1)}=1 / 2$ for each $u \in\left\{u_{c}, u_{d}\right\}$ and $\tau_{A}=(1 / 2,1 / 2)$.

Consider, specifically, the following equilibrium distribution $\tau=(1 / 2,0,0,1 / 2)$ where all players with conformist preferences choose action 0 and all players with nonconformist preferences choose actions 1 . We now show that there exists a sequence 
of finite-player games, with a number of players going to infinity, and a corresponding sequence of pure-strategy Nash equilibria such that the distribution over payoff functions and actions induced by the allocation of payoff functions and the Nash equilibrium converges to the equilibrium distribution $\tau$ of the non-atomic game.

To see the above, let $n \in \mathbb{N}, n \geq 4$ and even, and consider the $n$-player game where half of the players have payoff function $u_{d}$ and the remaining half of the players have payoff function $u_{c}^{n}$ defined by setting, for each $a \in A$ and $\pi \in \Delta$,

$$
u_{c}^{n}(a, \pi)= \begin{cases}u_{c}(0, \pi)+\frac{1}{n-1} & \text { if } a=0 \\ u_{d}(1, \pi) & \text { if } a=1\end{cases}
$$

It is then easy to see that the $n$-player game has a pure-strategy Nash equilibrium $f_{n}$ where all the players with payoff function $u_{c}^{n}$ play 0 and all the players with payoff function $u_{d}$ play 1 . Because $\left\langle u_{c}^{n}\right\rangle$ converges uniformly to $u_{c}$, the distribution over payoff functions and actions induced by the allocation of payoff functions and $f_{n}$ converges to the equilibrium distribution $\tau$ of the non-atomic game.

\section{Large Games}

In this section we introduce notation and terminology needed to set up our framework. It consists of the class of games where each player has a finite set of actions and a payoff function that depends on his choice and on the probability distribution on the set of actions induced by the choice of the other players.

\subsection{Finite-player anonymous games}

An anonymous game is defined as follows. There is a finite set $I=\{1, \ldots, n\}$ of players. For each $i \in I$, player $i$ 's action space is $A_{i}$, a nonempty subset of a finite action universe $A$. A pure strategy of player $i$ is an element $a_{i}$ of $A_{i}$. We denote a generic element of $\prod_{i \in I} A_{i}$ by $\tilde{a}$ and its $k$ th component by $\tilde{a}_{k}$. Given a profile $\tilde{a}=\left(\tilde{a}_{1}, \ldots, \tilde{a}_{n}\right)$ of pure strategies, and some pure strategy $a_{i}$ of player $i \in I$, we write $\left(a_{i}, \tilde{a}_{-i}\right)$ for $\left(\tilde{a}_{1}, \ldots, \tilde{a}_{i-1}, a_{i}, \tilde{a}_{i+1}, \ldots, \tilde{a}_{n}\right)$. 
For each $i \in I$, player $i$ 's payoff function depends on the probability distribution on the set of actions induced by the choice of the other players. The space of such distributions is

$$
E_{i}=\left\{\pi \in M(A): \pi=\frac{1}{n-1} \sum_{j \in I \backslash\{i\}} 1_{a_{j}} \text { where } a_{j} \in A_{j} \text { for all } j \in I \backslash\{i\}\right\},
$$

where $M(A)$ denotes the set of all probability measures on $A$; the set $M(A)$ is identified with the standard unit simplex of $\mathbb{R}^{\#(A)}$ and endowed with its standard norm. Also, in (1) and for each $a \in A, 1_{a}$ denotes the probability measure that assigns probability one to $\{a\}$.

Player $i$ 's payoff function is then given by $u_{i}: A_{i} \times E_{i} \rightarrow \mathbb{R}$, with $u_{i}(a, \pi)$ being player $i$ 's payoff when he plays action $a$ and faces the distribution $\pi$ on $A$ induced by the actions of all other players. The distribution on $A$ induced by an action profile $\tilde{a}_{-i}$ of all players other than $i$ is $\frac{1}{n-1} \sum_{j \in I \backslash\{i\}} 1_{\tilde{a}_{j}}$, i.e. it is the probability measure on $A$ with support $\left\{\tilde{a}_{j}: j \neq i\right\}$ such that, for each $a$ in its support,

$$
\frac{1}{n-1} \sum_{j \in I \backslash\{i\}} 1_{\tilde{a}_{j}}(a)=\frac{\left|\left\{j \neq i: \tilde{a}_{j}=a\right\}\right|}{n-1}
$$

is the fraction of players other than $i$ that play action $a$. Thus, given a pure strategy profile $\tilde{a}=\left(\tilde{a}_{1}, \ldots, \tilde{a}_{n}\right)$, the payoff of player $i$ is

$$
U_{i}(\tilde{a})=u_{i}\left(\tilde{a}_{i}, \frac{1}{n-1} \sum_{j \in I \backslash\{i\}} 1_{\tilde{a}_{j}}\right) .
$$

This concludes the definition of an anonymous game. In short, an anonymous game can be described by the set of players $I$ and the function $G$ assigning to each player his payoff function: $G(i)=u_{i}$ (player $i$ 's action set equals the projection on $A$ of the domain of $\left.u_{i}\right)$. We thus write $(I, G)$ for the game just defined.

A pure strategy profile $\tilde{a}=\left(\tilde{a}_{1}, \ldots, \tilde{a}_{n}\right) \in \prod_{i \in I} A_{i}$ of an anonymous game $(I, G)$ is a Nash equilibrium of $(I, G)$ if, for each $i \in I$ and $a_{i} \in A_{i}, U_{i}(\tilde{a}) \geq U_{i}\left(a_{i}, \tilde{a}_{-i}\right)$. It is a strict equilibrium of $(I, G)$ if, for each $i \in I$ and $a_{i} \in A_{i} \backslash\left\{\tilde{a}_{i}\right\}, U_{i}(\tilde{a})>U_{i}\left(a_{i}, \tilde{a}_{-i}\right)$.

Analogously to what we did regarding players' payoff functions, we often represent a pure strategy profile $\tilde{a}=\left(\tilde{a}_{1}, \ldots, \tilde{a}_{n}\right)$ as the function $f: I \rightarrow A$ defined by setting, for each $i \in I, f(i)=\tilde{a}_{i}$. 


\subsection{Space of games}

We let $\mathcal{U}$ denote the space of all continuous functions $u: A^{\prime} \times M^{\prime} \rightarrow \mathbb{R}$ where $A^{\prime}$ is a nonempty subset of $A$ and $M^{\prime}$ is a nonempty and closed subset of $M(A)$. The domain of $u \in \mathcal{U}$ is denoted by $\operatorname{dom}(u)$ and it is often written as $A_{u} \times M_{u}$. We endow $\mathcal{U}$ with a metric $d$ such that $u_{k} \rightarrow u$ in $\mathcal{U}$ if and only if (a) $\operatorname{dom}\left(u_{k}\right) \rightarrow \operatorname{dom}(u)$ in the Hausdorff metric topology and (b) $u_{k}\left(a_{k}, \pi_{k}\right) \rightarrow u(a, \pi)$ whenever $\left(a_{k}, \pi_{k}\right) \rightarrow(a, \pi)$, $(a, \pi) \in \operatorname{dom}(u)$ and $\left(a_{k}, \pi_{k}\right) \in \operatorname{dom}\left(u_{k}\right)$ for each $k \in \mathbb{N}$; see Section A.3.1 for details.

Each anonymous game $(I, G)$ defines a probability measure on $\nu_{(I, G)}$ on $\mathcal{U}$ defined by setting, for each Borel subset $U$ of $\mathcal{U}$,

$$
\nu_{(I, G)}(U)=\frac{\#(\{i \in I: G(i) \in U\})}{\#(I)} .
$$

Clearly, $\nu_{(I, G)}$ has a finite support.

We let the space of games be the set $\mathcal{M}$ of all Borel probability measures on $\mathcal{U}$ with compact support. We endow $\mathcal{M}$ with the topology such that $\nu_{n} \rightarrow \nu$ if both $\nu_{n} \rightarrow \nu$ in the narrow topology and $\operatorname{supp}\left(\nu_{n}\right) \rightarrow \operatorname{supp}(\nu)$ in the Hausdorff metric topology. ${ }^{6}$ The motivation for these choices is given in the following discussion and examples.

Our results concern sequences $\left\langle\left(I_{n}, G_{n}\right)\right\rangle_{n \in \mathbb{N}}$ of finite-player anonymous games with $\#\left(I_{n}\right) \rightarrow \infty$. They are obtained by studying the limit $\nu$ of the sequence of distributions $\left\langle\nu_{\left(I_{n}, G_{n}\right)}\right\rangle_{n \in \mathbb{N}}$. One aspect of our convergence notion is that whether or not $\left\langle\nu_{\left(I_{n}, G_{n}\right)}\right\rangle_{n \in \mathbb{N}}$ converges to $\nu$ depends only on players' payoffs at actions in their actions sets and action distributions that can actually occur in those games; this would not be true had we taken $\mathcal{U}$ to be the space of real-valued continuous functions on $A \times M(A)$.

The support of $\nu_{\left(I_{n}, G_{n}\right)}$ is finite for all $n$ but the support of $\nu$ need not be. We focus on distributions $\nu$ on $\mathcal{U}$ with compact support. This is standard: In fact, the assumption that the support of $\nu \in \mathcal{M}$ is compact is equivalent to the standard assumptions of a bounded and equicontinuous family of games (e.g. Rashid (1983),

\footnotetext{
${ }^{6}$ This two requirements mean that $\int_{\mathcal{U}} h \mathrm{~d} \nu_{n} \rightarrow \int_{\mathcal{U}} h \mathrm{~d} \nu$ for each continuous and bounded realvalued function $h$ on $\mathcal{U}$ and that the Hausdorff distance between $\operatorname{supp}\left(\nu_{n}\right)$ and $\operatorname{supp}(\nu)$ goes to zero.
} 
Carmona (2004) and Kalai (2004)). This is stated below in Lemma 1. In our setting, $U \subseteq \mathcal{U}$ is equicontinuous if, for each $\varepsilon>0$, there is $\delta>0$ such that $\left|u(a, \pi)-u\left(a, \pi^{\prime}\right)\right|<$ $\varepsilon$ whenever $u \in U, a \in A$ and $\pi, \pi^{\prime} \in M(A)$ are such that $(a, \pi),\left(a, \pi^{\prime}\right) \in \operatorname{dom}(u)$ and ||$\pi-\pi^{\prime}||<\delta$. Furthermore, $U$ is bounded if there is $B>0$ such that $|u(a, \pi)| \leq B$ whenever $u \in U, a \in A$ and $\pi \in M(A)$ are such that $(a, \pi) \in \operatorname{dom}(u)$.

Lemma 1. Let $U \subseteq \mathcal{U}$ be closed. Then $U$ is compact if and only if $U$ is bounded and equicontinuous.

One of the convergence requirement that we impose on sequences $\left\langle\left(I_{n}, G_{n}\right)\right\rangle_{n \in \mathbb{N}}$ is that $\nu_{\left(I_{n}, G_{n}\right)} \rightarrow \nu$ in the narrow topology. The narrow topology on $\mathcal{M}$ reflects the topology of the underlying space $\mathcal{U}$ of possible payoff functions in the sense of treating two distributions on $\mathcal{U}$ as close whenever they involve similar payoff functions with similar frequencies; e.g., if $\mu=\sum_{k=1}^{m} \alpha_{k} 1_{u_{k}}$ and $\mu^{\prime}=\sum_{k=1}^{m} \alpha_{k}^{\prime} 1_{u_{k}^{\prime}}$ are such that the payoff functions $u_{k}$ and $u_{k}^{\prime}$ are close in $\mathcal{U}$ for each $k$, and the frequencies $\alpha_{k}$ and $\alpha_{k}^{\prime}$ with which they occur are close for each $k$, then the distributions $\mu$ and $\mu^{\prime}$ are treated as close by the narrow topology.

The narrow topology also regards $\mu$ and $\mu^{\prime}$ as close when, for some $k, u_{k}$ and $u_{k}^{\prime}$ are not close in $\mathcal{U}$ provided that both $\alpha_{k}$ and $\alpha_{k}^{\prime}$ are sufficiently close to zero. This means that when two distributions are close according to the narrow topology, there may exist a set of payoff functions with small measure under one measure that are not close to any payoff function in the support of the other. This possibility is eliminated by requiring as we did in the definition of topology of $\mathcal{M}$ that $\operatorname{supp}\left(\nu_{n}\right) \rightarrow \operatorname{supp}(\nu)$ in the Hausdorff metric topology for $\nu_{n} \rightarrow \nu$.

As a result of the two requirements, every payoff function in the support of $\nu$ is close to a payoff function in the support of $\nu_{\left(I_{n}, G_{n}\right)}$ (and vice-versa) and their frequencies are also close.

\subsection{Non-atomic games}

Non-atomic games are described without specifying explicitly its set of players. Instead, only the distribution of players' payoff functions (and, implicity via their do- 
mains, of players' action sets) is specified. Roughly, a non-atomic game is $\nu \in \mathcal{M}$ such that every $u \in \operatorname{supp}(\nu)$ has the "right" domain as follows."

For each $u \in \mathcal{U}$, let the domain of $u$ be denoted by $A_{u} \times M_{u}$. Moreover, for each probability measure $\tau$ on $\mathcal{U} \times A$, let $\tau_{\mathcal{U}}$ be the marginal of $\tau$ on $\mathcal{U}$ and $\tau_{A}$ be the marginal of $\tau$ on $A$. Then, for each $\nu \in \mathcal{M}$, the set of action distributions that can arise is

$$
\begin{array}{r}
E(\nu)=\left\{\tau_{A}: \quad \tau \text { is a probability measure on } \mathcal{U} \times A\right. \text { such that } \\
\\
\left.\tau_{\mathcal{U}}=\nu \text { and }(u, a) \in \operatorname{supp}(\tau) \text { implies } a \in A_{u}\right\} .
\end{array}
$$

Note that $M_{u}, u \in \operatorname{supp}(\nu)$, plays no role in the definition of $E(\nu)$. Formally, a non-atomic game is $\nu \in \mathcal{M}$ such that $M_{u}=E(\nu)$ for each $u \in \operatorname{supp}(\nu){ }^{8}$

An equilibrium distribution of $\nu$ is a Borel probability measure $\tau$ on $\mathcal{U} \times A$ such that $\tau_{\mathcal{U}}=\nu$ and

$$
\tau\left(\left\{(u, a) \in \mathcal{U} \times A: a \in A_{u} \text { and } u\left(a, \tau_{A}\right) \geq u\left(a^{\prime}, \tau_{A}\right) \text { for all } a^{\prime} \in A_{u}\right\}\right)=1 .
$$

Letting, for each $u \in \operatorname{supp}(\nu)$ and $\pi \in M_{u}$,

$$
\varphi(u, \pi)=\left\{a \in A_{u}: u(a, \pi)=\max _{a^{\prime} \in A_{u}} u\left(a^{\prime}, \pi\right)\right\}
$$

the second requirement in the definition of an equilibrium distribution can be written as $\operatorname{supp}(\tau) \subseteq\left\{(u, a) \in \mathcal{U} \times A: a \in \varphi\left(u, \tau_{A}\right)\right\}$.

We use $\mathcal{G}$ to denote the set of all non-atomic games and give $\mathcal{G}$ the subspace topology defined from that of $\mathcal{M}$.

\footnotetext{
${ }^{7}$ Such games are also known as large distributionalized games as in e.g. Khan, Rath, Yu, and Zhang (2017).

${ }^{8}$ For example, if $A=\{0,1\}, \nu\left(\left\{u \in \mathcal{U}: A_{u}=\{0\}\right\}\right)=1 / 3$ and $\nu\left(\left\{u \in \mathcal{U}: A_{u}=\{0,1\}\right\}\right)=$ $2 / 3$, then $E(\nu)=\left\{\pi \in M(A): \pi_{0} \geq 1 / 3\right\}$. Thus, the requirement that $M_{u}=E(\nu)$ for each $u \in \operatorname{supp}(\nu)$ means that payoff functions have a "minimal domain": Payoffs are defined only for the action distributions that can arise. Conversely, $E(\nu) \subset M_{u}$ means that $u$ has been extended beyond what is needed to analyze the game - but such extension is not needed and is, in this sense, arbitrary. For this reason, as pointed out before, we want the convergence of $\left\langle\nu_{\left(I_{n}, G_{n}\right)}\right\rangle_{n \in \mathbb{N}}$ to $\nu$ to depends only on players' payoffs at actions in their actions sets and action distributions that can actually occur in those games.
} 


\section{Generic existence of pure strategy Nash equilib- ria for finite action games}

Example 1 shows that not all sufficiently large finite-player games have a pure strategy Nash equilibria. More precisely, using the notation of Section 4, it shows that there exists $\nu \in \mathcal{G}$ and a sequence of finite-player games $\left\langle\left(I_{n}, G_{n}\right)\right\rangle_{n \in \mathbb{N}}$ with $\nu_{\left(I_{n}, G_{n}\right)} \rightarrow \nu$ and $\#\left(I_{n}\right) \rightarrow \infty$ such that, for each $N \in \mathbb{N}$, there exists $n \geq N$ such that $\left(I_{n}, G_{n}\right)$ has no pure strategy Nash equilibrium.

The above conclusion requires the choice of a special $\nu$, namely $\nu\left(\left\{u_{c}\right\}\right)=1 / 2=$ $\nu\left(\left\{u_{d}\right\}\right)$ in Example 1. Indeed, there are distributions $\mu \in \mathcal{G}$ arbitrarily close to $\nu$ such that each finite-player game $(I, G)$ with $\nu_{(I, G)}$ sufficiently close to $\mu$ and \#(I) sufficiently large has a pure strategy Nash equilibrium. This has been illustrated in Example 2 by perturbing the function $u_{d}$. Alternatively, this can be seen, for instance, by letting $\mu\left(\left\{u_{c}\right\}\right)=\theta \in \mathbb{Q} \cap(1 / 2,1 / 2+\eta)$ (where $\eta>0$ is arbitrarily small), $\mu\left(\left\{u_{d}\right\}\right)=1-\theta$ and by considering, in the game $(I, G)$ with $\#(I)=n$ and $\nu_{(I, G)}=$ $\sum_{u \in \operatorname{supp}(\mu)} \mu(u) 1_{\left.u\right|_{A \times E_{n}}}$ (where $E_{n}=\left\{\pi \in M(A): \pi_{0}=k /(n-1)\right.$ for some $k=$ $0, \ldots, n-1\})$, the pure strategy profile $a^{*}$ defined by setting $a_{i}^{*}=0$ if $G(i)=\left.u_{c}\right|_{A \times E_{n}}$

and $a_{i}^{*}=1$ if $G(i)=\left.u_{d}\right|_{A \times E_{n}}$. It is easy to see that this strategy profile is a Nash equilibrium (in fact, a strict equilibrium) of $(I, G)$ provided that $\theta-\frac{1}{n}>\frac{1}{2}$, i.e. that $\#(I)$ is sufficiently large.

This discussion suggests that sufficiently large games whose distribution is sufficiently close to a generic non-atomic game have pure strategy Nash equilibria. Theorem 1 shows that this is indeed the case, thus answering the first question we posed in the introduction.

Theorem 1. Suppose that $A$ is finite. Then there is an open and dense subset $\mathcal{G}^{*}$ of $\mathcal{G}$ such that if $\nu \in \mathcal{G}^{*}$ and $\left\langle\left(I_{n}, G_{n}\right)\right\rangle_{n \in \mathbb{N}}$ is a sequence of games with $\nu_{\left(I_{n}, G_{n}\right)} \rightarrow \nu$ and $\#\left(I_{n}\right) \rightarrow \infty$, then there is $N \in \mathbb{N}$ such that $\left(I_{n}, G_{n}\right)$ has a strict equilibrium whenever $\#\left(I_{n}\right) \geq N$.

Equivalently, Theorem 1 says that sufficiently large games whose distribution is 
sufficiently close to a generic non-atomic game have strict Nash equilibria. Formally: For each $\nu \in \mathcal{G}^{*}$, there exists an open neighborhood $O$ of $\nu$ and $N \in \mathbb{N}$ such that each finite-player game $(I, G)$ with $\nu_{(I, G)} \in O$ and $\#\left(I_{n}\right) \geq N$ has a strict equilibrium.

The idea of the proof of Theorem 1 is as follows. Let $\mathcal{G}^{*}$ be the subset of $\mathcal{G}$ consisting of those $\nu$ that have a strict equilibrium distribution, i.e. some equilibrium distribution $\tau$ such that $\#\left(\varphi\left(u, \tau_{A}\right)\right)=1$ for each $u \in \operatorname{supp}(\nu)$. Such strict equilibrium distribution is then used to construct strict equilibria for sequences $\left\langle\left(I_{n}, G_{n}\right)\right\rangle_{n \in \mathbb{N}}$ of finite-player games with $\nu_{\left(I_{n}, G_{n}\right)} \rightarrow \nu$ and $\#\left(I_{n}\right) \rightarrow \infty$. The set $\mathcal{G}^{*}$ is open in $\mathcal{G}$ for a similar reason, namely the strict equilibrium distribution of $\nu$ is used to construct strict equilibria of non-atomic games $\nu^{\prime}$ with $\nu^{\prime}$ close to $\nu$. Furthermore, $\mathcal{G}^{*}$ is dense in $\mathcal{G}$ since, given an equilibrium distribution $\tau$ of $\nu$, the payoff functions in $\operatorname{supp}(\nu)$ can be slightly perturbed to create a non-atomic game $\nu^{\prime}$ close to $\nu$ and to create a strict equilibrium $\tau^{\prime}$ of $\nu^{\prime}$.

\section{Stability of equilibria of non-atomic games}

Two issues regarding the stability of equilibrium distributions of non-atomic games are considered in this section: Their asymptotic implementation and the generic existence of robust equilibrium distributions.

\subsection{Asymptotic implementation}

A classical issue regarding large games is whether or not the equilibrium behavior of non-atomic games provides a good approximation of the equilibrium behavior of similar large finite-player games. Indeed, non-atomic games are a tractable idealization of large finite-player games and, thus, an affirmative answer to this question is needed to justify them as an useful idealization. It turns out that the tools we have developed to analyze the generic existence of pure strategy Nash equilibria of large finite-player games are also useful to address this question, as we show in this section.

As discussed in the introduction, existing results show that equilibria of non- 
atomic games are limits of pure $\varepsilon$-equilibria of finite-player games. ${ }^{9}$ Can we obtain the same conclusion with pure strategy Nash equilibria? The importance of this question arises because $\varepsilon$-equilibria of large finite-player games are not necessarily appealing, as it can be seen using the example discussed in the introduction.

Example 4. Let $A=\{0,1\}, n \in \mathbb{N}$ and $u_{n}(a, \pi)=a \frac{a+(n-1) \pi_{1}}{n}$ for each $a \in A$ and $\pi \in$ $M(A)$. Consider the finite-player game $\left(I_{n}, G_{n}\right)$ with $\#\left(I_{n}\right)=n$ and $\left.G_{n} \equiv u_{n}\right|_{A \times E_{n}}$ where $E_{n}=\left\{\pi \in M(A): \pi_{1}=k /(n-1)\right.$ for some $\left.k=0, \ldots, n-1\right\}$. It is easy to see that the pure strategy profile where each player chooses 0 is an $\varepsilon$-equilibrium of $\left(I_{n}, G_{n}\right)$ with $\varepsilon=1 / n$. In contrast, $\left(I_{n}, G_{n}\right)$ has a unique, strictly dominant, Nash equilibrium, namely $a^{*}$ with $a_{i}^{*}=1$ for all $i \in I_{n}$.

Based on the above discussion, at the very least, we would like to have the existence sequences of finite-player games converging to a given non-atomic game and having pure strategy Nash equilibria whose distributions converge to a given equilibrium distribution of the non-atomic game. Formally, let $\nu \in \mathcal{G}$ and let $\tau$ be an equilibrium distribution of the non-atomic game $\nu$. We say that $\tau$ is asymptotically implementable if there exists a sequence $\left\langle\left(I_{n}, G_{n}\right)\right\rangle_{n \in \mathbb{N}}$ of finite-player games such that $\#\left(I_{n}\right) \rightarrow \infty$, $\nu_{\left(I_{n}, G_{n}\right)} \rightarrow \nu$ and, for all $n$ larger than some $N \in \mathbb{N},\left(I_{n}, G_{n}\right)$ has a pure strategy Nash equilibrium $f_{n}$ such that the sequence of distributions of the map $\left(G_{n}, f_{n}\right)$ converges to $\tau$ narrowly. ${ }^{10}$

Theorem 2 shows that this property holds in general.

Theorem 2. Suppose that $A$ is finite. Then every equilibrium distribution $\tau$ of every non-atomic game $\nu \in \mathcal{G}$ is asymptotically implementable.

Theorem 2 answers the second question we posed in the introduction by showing that equilibrium distributions of non-atomic games are limits of Nash equilibria

\footnotetext{
${ }^{9} \mathrm{~A}$ pure strategy profile $a^{*}=\left(a_{1}^{*}, \ldots, a_{n}^{*}\right)$ of an anonymous game $(I, G)$ is an $\varepsilon$-equilibrium of $(I, G), \varepsilon>0$, if for each $i \in I$ and $a_{i} \in A_{i}, U_{i}\left(a^{*}\right) \geq U_{i}\left(a_{i}, a_{-i}^{*}\right)-\varepsilon$.

${ }^{10}$ For each $n \in \mathbb{N}$, the distribution of the map $\left(G_{n}, f_{n}\right)$ is the probability measure on $\mathcal{U} \times A$ that assigns probability $\frac{\#\left(\left\{i \in I_{n}:\left(G_{n}(i), f_{n}(i)\right)=(u, a)\right\}\right)}{n}$ to each $(u, a) \in G_{n}\left(I_{n}\right) \times f_{n}\left(I_{n}\right)$.
} 
of large finite games. It provides a justification for equilibrium distributions of nonatomic games, namely that each such distribution is arbitrarily close to the distribution induced by a pure strategy Nash equilibrium of a large finite-player game. This property holds for each non-atomic game, not just for generic ones.

The idea for the proof of Theorem 2 is as follows. First, as already noted, given an equilibrium distribution $\tau$ of a non-atomic game $\nu$, the payoff functions in $\operatorname{supp}(\nu)$ can be slightly perturbed to create a non-atomic game $\nu^{\prime}$ close to $\nu$ and to create a strict equilibrium $\tau^{\prime}$ of $\nu^{\prime}$. We can then obtain a sequence of finite-player games converging to $\nu^{\prime}$ and extend the logic of Theorem 1 to get a corresponding sequence of pure strategy Nash equilibria of these finite-player games whose distributions converge to $\tau^{\prime}$. Making $\tau^{\prime}$ converge to $\tau$ completes the argument.

\subsection{Robustness}

Our results so far allow us also to define a more robust equilibrium concept for nonatomic games and to obtain its generic existence.

Such equilibrium concept is obtained by requiring, for each sequence of finiteplayer games converging to a given non-atomic game, the existence of a corresponding sequence of pure strategy Nash equilibria whose distributions converge to a given equilibrium distribution of the non-atomic game. Formally, let $\nu \in \mathcal{G}$ and let $\tau$ be an equilibrium distribution of the non-atomic game $\nu$. We say that $\tau$ is asymptotically robust if for each sequence $\left\langle\left(I_{n}, G_{n}\right)\right\rangle_{n \in \mathbb{N}}$ of finite-player games such that $\#\left(I_{n}\right) \rightarrow \infty$ and $\nu_{\left(I_{n}, G_{n}\right)} \rightarrow \nu$, there exists $N \in \mathbb{N}$ such that, for all $n \geq N,\left(I_{n}, G_{n}\right)$ has a pure strategy Nash equilibrium $f_{n}$ such that the sequence of distributions of the map $\left(G_{n}, f_{n}\right)$ converges to $\tau$ narrowly.

It is not possible to obtain the existence of an asymptotically robust equilibrium distribution for each non-atomic game $\nu \in \mathcal{G}$. This can be seem by letting $A=\{0,1\}$ and $u: A \times M(A) \rightarrow \mathbb{R}$ such that $u(a, \pi)=\pi_{1}$ for each $a \in A$ and $\pi \in M(A)$ and a non-atomic game $\nu$ be such that $\nu(\{u\})=1$. By considering the sequence $\left\langle\left(I_{n}, G_{n}\right)\right\rangle_{n \in \mathbb{N}}$ with $\#\left(I_{n}\right)=n$ and $G_{n} \equiv u_{n}: A \times E_{n} \rightarrow \mathbb{R}$ where $u_{n}(a, \pi)=\frac{a+(n-1) \pi_{1}}{n}$ for each $a \in A$ and $\pi \in E_{n}=\left\{\pi \in M(A): \pi_{1}=k /(n-1)\right.$ for some $\left.k=0, \ldots, n-1\right\}$, 
it is clear that the only equilibrium distribution $\tau$ of $\nu$ that can satisfy the requirements of asymptotic robustness is such that $\tau_{A}=1_{1}$. But consider, for each $n \in \mathbb{N}$, $\hat{u}_{n}: A \times E_{n} \rightarrow \mathbb{R}$ defined by $\hat{u}_{n}(1, \pi)=\frac{1 / 2+(n-1) \pi_{1}}{n}$ and $\hat{u}_{n}(0, \pi)=\frac{1+(n-1) \pi_{1}}{n}$ for each

$\pi \in M(A)$ and $\left(I_{n}, \hat{G}_{n}\right)$. Then, $\nu_{\left(I_{n}, \hat{G}_{n}\right)} \rightarrow \nu$ and 0 is a dominant strategy for each player in $\left(I_{n}, \hat{G}_{n}\right)$.

Nevertheless, Theorem 3 below establishes the generic existence of asymptotically robust equilibrium distributions.

Theorem 3. Suppose that $A$ is finite. Then there exists an open and dense subset $\mathcal{G}^{*}$ of $\mathcal{G}$ such that each $\nu \in \mathcal{G}^{*}$ has an asymptotically robust equilibrium distribution.

Theorem 3 is a corollary of some of the arguments used in the proof of Theorems 1 and 2 . The set $\mathcal{G}^{*}$ is the same as in Theorem 1 , namely the subset of $\mathcal{G}$ consisting of those $\nu$ such that the non-atomic game with distribution $\nu$ has a strict equilibrium distribution. The proof of Theorem 2 then shows that, given $\nu \in \mathcal{G}^{*}$ and a strict equilibrium distribution $\tau$ of $\nu$, each sequence of finite-player games converging to $\nu$ has a corresponding sequence of pure strategy Nash equilibria whose distributions converge to $\tau$. Thus, $\tau$ is asymptotically robust.

\section{General compact metric action spaces}

In this section we extend our results by allowing players' action spaces to be general compact metric spaces. Thus, we assume in this section that players' action universe $A$ is a compact metric space.

The space $M(A)$ is now the space of Borel probability measures on $A$ endowed with the narrow topology. The space $\mathcal{U}$ is now the space of all continuous functions $u: A^{\prime} \times M^{\prime} \rightarrow \mathbb{R}$ where $A^{\prime}$ is a nonempty and closed subset of $A$ and $M^{\prime}$ is a nonempty and closed subset of $M(A)$; it is endowed with metric $d$ such that, as before, $u_{k} \rightarrow u$ in $\mathcal{U}$ if and only if (a) $\operatorname{dom}\left(u_{k}\right) \rightarrow \operatorname{dom}(u)$ in the Hausdorff metric topology and (b) $u_{k}\left(a_{k}, \pi_{k}\right) \rightarrow u(a, \pi)$ whenever $\left(a_{k}, \pi_{k}\right) \rightarrow(a, \pi),(a, \pi) \in \operatorname{dom}(u)$ and $\left(a_{k}, \pi_{k}\right) \in \operatorname{dom}\left(u_{k}\right)$ for each $k \in \mathbb{N}$ 
An anonymous game is still defined by a finite set of players $I=\{1, \ldots, n\}$ and a function $G: I \rightarrow \mathcal{U}$ assigning payoff functions to players, but where $\mathcal{U}$ is as in the above paragraph.

We use mixed strategy Nash equilibria of large finite-player games in the extension of our results to the case of a general compact metric action universe. To give some intuition for the difficulties involved in this extension and for why we allow for mixed strategies in the generic case, consider a non-atomic game $\nu$ where all players have the same singleton set, e.g. $\{1 / 2\}$, as action space. Such game has a unique equilibrium distribution which is trivially strict. Thus, when the universal action space $A$ is finite, $\nu \in \mathcal{G}^{*}$; consequently, any non-atomic game $\nu^{\prime}$ sufficiently close to $\nu$ has a strict equilibrium and any finite-player game $(I, G)$ with $\nu_{(I, G)}$ sufficiently close to $\nu$ and \#(I) sufficiently large has a strict equilibrium. However, these two conclusions fail when $A$ contains an open neighborhood of $1 / 2$, e.g. when $A=[0,1]$. For instance, consider games (non-atomic or with finitely many players) in which (i) each player has $\left\{1 / 2-\delta_{n}, 1 / 2+\delta_{n}\right\}$ as action space, (ii) $\delta_{n} \rightarrow 0$, (iii) half of the players have payoff function $u_{n, c}$, defined as the restriction of $(a, \pi) \mapsto a\left(\int_{A} a^{\prime} \mathrm{d} \pi\left(a^{\prime}\right)-\frac{1}{2}\right)$ to the appropriate domain and (iv) half of the players have payoff function $u_{n, d}=-u_{n, c}$. It is then easy to see that any such non-atomic game has no strict equilibrium distribution (indeed, any equilibrium distribution $\tau$ is such that $\int_{A} a \mathrm{~d} \tau_{A}(a)=1 / 2$ and, hence, $\varphi\left(u, \tau_{A}\right)=A_{u}$ for each $\left.u \in\left\{u_{n, c}, u_{n, d}\right\}\right)$. It is also easy to see that, analogously to Example 1, any such finite-player game with an even number of players has no pure strategy Nash equilibrium.

We will shortly return to the above example to see how mixed strategies that are "nearly pure" are helpful. A mixed strategy of player $i$ is an element $\sigma_{i}$ of $M\left(A_{i}\right)$, where $A_{i}=A_{G(i)}$ (recall that the domain of $u \in \mathcal{U}$ is denoted by $A_{u} \times M_{u}$ ). Given a profile $\sigma=\left(\sigma_{1}, \ldots, \sigma_{n}\right)$ of mixed strategies, and some mixed strategy $\sigma_{i}^{\prime}$ of player $i \in I$, we write $\left(\sigma_{i}^{\prime}, \sigma_{-i}\right)$ for $\left(\sigma_{1}, \ldots, \sigma_{i-1}, \sigma_{i}^{\prime}, \sigma_{i+1}, \ldots, \sigma_{n}\right)$. Furthermore, we let $\tilde{\sigma}=$ $\sigma_{1} \otimes \cdots \otimes \sigma_{n}$, i.e., $\tilde{\sigma}$ is the product probability measure on $\prod_{i \in I} A_{i}$ defined from the measures $\sigma_{1}, \ldots, \sigma_{n}$.

Given a mixed strategy profile $\sigma=\left(\sigma_{1}, \ldots, \sigma_{n}\right)$, the expected payoff of player 
$i \in I$ is

$$
U_{i}(\sigma)=\int_{\prod_{j \in I} A_{j}} u_{i}\left(\tilde{a}_{i}, \frac{1}{n-1} \sum_{j \in I \backslash\{i\}} 1_{\tilde{a}_{j}}\right) \mathrm{d} \tilde{\sigma}(\tilde{a})
$$

where $u_{i}=G(i)$. A mixed strategy profile $\sigma=\left(\sigma_{1}, \ldots, \sigma_{n}\right)$ of an anonymous game $(I, G)$ is a Nash equilibrium of $(I, G)$ if, for each $i \in I$ and $a_{i} \in A_{i}, U_{i}(\sigma) \geq U_{i}\left(a_{i}, \sigma_{-i}\right)$.

Returning to the above example, it is easy to see that, in a finite-player game $\left(I_{n}, G_{n}\right)$ satisfying (i)-(iv), $\sigma^{n}$ such that $\sigma_{i}^{n}=\left(1_{1 / 2-\delta_{n}}+1_{1 / 2+\delta_{n}}\right) / 2$ for each $i \in I_{n}$ is a Nash equilibrium of $\left(I_{n}, G_{n}\right)$ for each $n \in \mathbb{N}$, since

$$
U_{i}\left(a_{i}, \sigma_{-i}^{n}\right)=a_{i}\left(\sum_{j \neq i} \frac{\delta_{n}}{n-1}\left(\sigma_{j}^{n}\left(1 / 2+\delta_{n}\right)-\sigma_{j}^{n}\left(1 / 2-\delta_{n}\right)\right)\right)=0
$$

for each $a \in A_{n}$ and $i \in I_{n}$. This Nash equilibrium $\sigma^{n}$ of $\left(I_{n}, G_{n}\right)$ is "nearly pure" in the sense that the support of $\sigma_{i}^{n}, i \in I_{n}$, is contained in a small neighborhood of a pure strategy, namely the closed ball of radius $\delta_{n}$ of $1 / 2$. Thus, by making $n$ sufficiently large, it becomes nearly impossible to distinguish $\sigma^{n}$ from a pure strategy profile.

Our next result shows that the above is true generically in the entire class of games that we consider in this section. Given $\varepsilon>0$, we say that a mixed strategy profile $\sigma=\left(\sigma_{1}, \ldots, \sigma_{n}\right)$ of an anonymous game $(I, G)$ is $\varepsilon$-concentrated if, for each $i \in I$, diameter $\left(\operatorname{supp}\left(\sigma_{i}\right)\right) \leq \varepsilon$. Thus, in an $\varepsilon$-concentrated strategy profile $\sigma$ and given $a_{i} \in \operatorname{supp}\left(\sigma_{i}\right)$ for each $i \in I$, there is a zero probability of a pure strategy profile occurring in which some player plays an action whose distance is more than $\varepsilon$ from $a_{i}$

Our extension of Theorem 1 is as follows.

Theorem 4. Suppose that $A$ is a compact metric space. Then, for each $\varepsilon>0$, there is an open dense subset $\mathcal{G}_{\varepsilon}^{*}$ of $\mathcal{G}$ such that if $\nu \in \mathcal{G}_{\varepsilon}^{*}$ and $\left\langle\left(I_{n}, G_{n}\right)\right\rangle_{n \in \mathbb{N}}$ is a sequence of games with $\nu_{\left(I_{n}, G_{n}\right)} \rightarrow \nu$ and $\#\left(I_{n}\right) \rightarrow \infty$, then there is an $N \in \mathbb{N}$ such that whenever $\#\left(I_{n}\right) \geq N,\left(I_{n}, G_{n}\right)$ has a mixed strategy Nash equilibrium $\sigma$ such that $\sigma$ is $\varepsilon$-concentrated. 
Indeed, Theorem 4 extends Theorem 1 because, when $A$ is finite and $\varepsilon$ is sufficiently small, any $\varepsilon$-concentrated strategy profile is pure.

The idea of the proof of Theorem 4 is similar to that of Theorem 1 but more involved. In particular, the generic set $\mathcal{G}^{*}$ consists roughly of the set of those $\nu \in \mathcal{G}$ that have a continuous symmetric strategy, i.e. a continuous $s: \operatorname{supp}(\nu) \rightarrow A$, such that, for each $u \in \operatorname{supp}(\nu)$, no action outside a small neighborhood of $s(u)$ belongs to $\varphi\left(u, \nu \circ s^{-1}\right) \cdot{ }^{11}$

We turn now to the asymptotic implementation of the equilibrium distributions of non-atomic games, which we show to hold exactly as in Theorem 2. This is possible because, given an equilibrium distribution $\tau$ of a non-atomic game $\nu$, the payoff functions in $\operatorname{supp}(\nu)$ can be slightly perturbed to create a generic non-atomic game $\nu^{\prime}$ close to $\nu$ and to create a continuous symmetric equilibrium $\tau^{\prime}$ of $\nu^{\prime}$. We can then obtain a sequence of finite-player games converging to $\nu^{\prime}$ and get a corresponding sequence of $\varepsilon$-concentrated mixed strategy Nash equilibria of these finite-player games whose distributions converge to $\tau^{\prime}$. By ensuring that, in the construction of $\nu^{\prime}$ and of the finite-player games, the "potentially optimal" actions (i.e. those in a small neighborhood of $s(u)$ for each $\left.u \in \operatorname{supp}\left(\nu^{\prime}\right)\right)$ are isolated, it follows that each $\varepsilon$-concentrated mixed strategy Nash equilibrium is pure; in this way, we obtain a sequence of pure strategy Nash equilibria of the finite-player games whose distributions converge to $\tau^{\prime}$. Then making $\tau^{\prime}$ converge to $\tau$ completes the argument.

Theorem 5. Suppose that $A$ is a compact metric space. Then every equilibrium distribution $\tau$ of every non-atomic game $\nu \in \mathcal{G}$ is asymptotically implementable.

\section{Applications}

We apply our results to two economic problems based on Azevedo and Budish (2019). They consist of the allocation, to a large number of individuals and through an anonymous mechanism, of indivisible objects and schools. In both cases, we consider the

\footnotetext{
${ }^{11}$ The distribution $\nu \circ s^{-1}$ over actions is defined by setting, for each Borel $B \subseteq A, \nu \circ s^{-1}(B)=$ $\nu(\{u \in \operatorname{supp}(\nu): s(u) \in B\})$. For the notion of a symmetric equilibrium see Mas-Colell (1984).
} 
case where individual preferences are unrestricted (beyond the assumption that each individual's payoff depends continuously only on his own action and on the distribution of actions chosen by the other individuals). In addition, for the allocation of an indivisible object, we consider the case where individual preferences are restricted to reflect the use of a specific mechanism, namely that of the first-price auction.

\subsection{Allocation of indivisible objects}

Consider the replication of the standard case where one object is allocated to one of $k>1$ individuals. Thus, there are $n$ units of an object to be allocated between $n k$ individuals where $n \in \mathbb{N}$. Each individual $i \in I_{n}=\{1, \ldots, n k\}$ desires at most one unit of the object and makes a bid. Bids are discrete, non-negative and bounded above. Thus, let $A=\{0,1, \ldots, \bar{a}\}$ where $\bar{a} \in \mathbb{N} \backslash\{0\}$ and $A_{i}=A$ for each $i \in I_{n}$. The allocation of the $n$ units of the object is done anonymously so that each individual's allocation depends only on his own bid and on the distribution of bids made by the other individuals. Thus, letting $\tilde{a}=\left(\tilde{a}_{1}, \ldots, \tilde{a}_{n k}\right)$ be a profile of bids, we let $i$ 's payoff be

$$
u_{i}\left(\tilde{a}_{i}, \frac{1}{n k-1} \sum_{j \in I_{n} \backslash\{i\}} 1_{\tilde{a}_{j}}\right) .
$$

In this formalization, each individual's payoff function is, apart from the continuity and anonymity requirements, unrestricted and, therefore, can depend on his own bid and on the distribution of the bids of the other players in any arbitrary way. This means that we are not restricting attention to a specific allocation mechanism, which would describe whether or not an individual receives one unit of the object (or, more generally, the probability of receiving the object) as a function of the bid profile. Moreover, this formalization also allows for payoff functions that are not obtained from preferences over the probability of consuming the object and the outcome of the allocation mechanism. This gives a very general formalization of the problem, which has the advantage that Theorems 1 and 2 apply to it. 


\subsection{First-price auctions}

Instead of allowing for the generality of the previous section, one may prefer to focus on the case where a specific allocation mechanism is used and where individual preferences are obtained from preferences over the probability of consuming the object and the outcome of the allocation mechanism. In this case, not all functions in $\mathcal{U}$ can arise as some player's payoff function and, thus, our results need to be specialized to the class of games having payoff functions that can arise.

We illustrate this issue for the case of first-price auctions. For each $n \in \mathbb{N}$, each individual $i \in I_{n}$ has a valuation $v_{i} \in \mathbb{R}_{+}$for the object, so that he derives a payoff of $v_{i}$ from the consumption of one unit of the good and zero otherwise. The first-price auction works as follows. Given $\pi \in M(A)$, let the market-clearing price be

$$
p^{*}(\pi)=\max \left\{p \in A: \sum_{p^{\prime} \geq p} \pi\left(p^{\prime}\right) \geq \frac{1}{k}\right\} ;
$$

in words, $p^{*}(\pi)$ is the highest price at which demand exceed supply. Indeed, for each $n \in \mathbb{N}$ and action profile $\tilde{a}=\left(\tilde{a}_{1}, \ldots, \tilde{a}_{n k}\right), \sum_{p^{\prime} \geq p} \pi\left(p^{\prime}\right) \geq \frac{1}{k}$ is equivalent to $\#(\{i \in$ $\left.\left.I_{n}: \tilde{a}_{i} \geq p\right\}\right) \geq n$ when $\pi(p)=\frac{\#\left(\left\{i \in I_{n}: \tilde{a}_{i}=p\right\}\right)}{n k}$ for each $p \in A$. The probability that each player obtains one unit of the object when his action is $a \in A$ and the distribution of actions is $\pi$ is

$$
\Phi(a, \pi)= \begin{cases}1 & \text { if } a>p^{*}(\pi), \\ \frac{\frac{1}{k}-\sum_{p>p^{*}(\pi)} \pi(p)}{\pi\left(p^{*}(\pi)\right)} & \text { if } a=p^{*}(\pi), \\ 0 & \text { if } a<p^{*}(\pi)\end{cases}
$$

For each $n \in \mathbb{N}$ and bid profile $\tilde{a}=\left(\tilde{a}_{1}, \ldots, \tilde{a}_{n k}\right)$, player $i$ 's payoff is then

$$
u_{i}\left(\tilde{a}_{i}, \frac{1}{n k-1} \sum_{j \in I_{n} \backslash\{i\}} 1_{\tilde{a}_{j}}\right)=\Phi\left(\tilde{a}_{i}, \frac{1}{n k} \sum_{j=1}^{n k} 1_{\tilde{a}_{j}}\right)\left(v_{i}-\tilde{a}_{i}\right) \cdot{ }^{12}
$$

We will keep the allocation mechanism fixed and, thus, players' payoff are determined by their valuations. Hence, in the context of this section, a game is probability

${ }^{12}$ Note that $\frac{\sum_{j=1}^{n k} 1_{\tilde{a}_{j}}}{n k}=\frac{1_{\tilde{a}_{i}}}{n k}+\frac{n k-1}{n k} \frac{\sum_{j \neq i} 1_{\tilde{a}_{j}}}{n k-1}$ for each $i \in I_{n}$; hence, for each player $i, p^{*}, \Phi$ and $i$ 's payoff depends only on $i$ 's action and on the distribution of actions chosen by the players other than $i$. 
measure $\nu$ on $\mathbb{R}_{+}$with compact support. We let this set be denoted by $\tilde{\mathcal{G}}$ and, as in Section 4.2, endowed it with the topology such that $\nu_{n} \rightarrow \nu$ if both $\nu_{n} \rightarrow \nu$ in the narrow topology and $\operatorname{supp}\left(\nu_{n}\right) \rightarrow \operatorname{supp}(\nu)$ in the Hausdorff metric topology. Finiteplayer games are as in Section 4.1 except that now $G$ maps the set of players $I$ into $\mathbb{R}_{+}$.

A difficulty with this setting is that all bids below the market-clearing price yield a zero payoff and are, therefore, indifferent. This prevents us from obtaining the (generic, asymptotic) existence of strict equilibria. But, apart from this issue, Theorems 1 and 2 extend to this setting.

Theorem 6. There is an open and dense subset $\tilde{\mathcal{G}}^{*}$ of $\tilde{\mathcal{G}}$ such that if $\nu \in \tilde{\mathcal{G}}^{*}$ and $\left\langle\left(I_{n}, G_{n}\right)\right\rangle_{n \in \mathbb{N}}$ is a sequence of games with $\nu_{\left(I_{n}, G_{n}\right)} \rightarrow \nu$ and $\#\left(I_{n}\right) \rightarrow \infty$, then there is $N \in \mathbb{N}$ such that $\left(I_{n}, G_{n}\right)$ has a pure-strategy Nash equilibrium whenever $\#\left(I_{n}\right) \geq N$.

Theorem 7. Every equilibrium distribution $\tau$ of every non-atomic game $\nu \in \tilde{\mathcal{G}}$ is asymptotically implementable.

The idea of the proof of Theorems 6 and 7 is analogous to that of Theorems 1 and 2 with the following important differences. First, it is now impossible to make certain actions be strict best-replies, namely those actions below the market-clearing price. This issue arise because of the narrow set of payoff functions allowed, which also complicates the denseness part of the argument. A final complication arises because players' payoff functions are not continuous as it can be seen by considering, when $\bar{a} \geq 2, v=3, \pi=\left(\frac{1}{k}\right) 1_{2}+\left(\frac{k-1}{k}\right) 1_{0}$ and $\pi_{j}=\left(\frac{1}{k}-\frac{1}{j}\right) 1_{2}+\left(\frac{k-1}{k}+\frac{1}{j}\right) 1_{0}$ for each $j \in$ $\mathbb{N} \backslash\{0\}$; then $\pi_{j} \rightarrow \pi, p^{*}(\pi)=2, p^{*}\left(\pi_{j}\right)=0, \Phi(1, \pi)(v-1)=0$ and $\Phi\left(1, \pi_{j}\right)(v-1)=2$ for each $j \in \mathbb{N} \backslash\{0\}$.

The above difficulties are avoided by letting $\tilde{\mathcal{G}}^{*}$ be the subset of $\tilde{\mathcal{G}}$ consisting of those $\nu$ that have an "essentially strict equilibrium distribution", i.e. some equilibrium distribution $\tau$ such that $\sum_{p \geq p^{*}\left(\tau_{A}\right)} \tau_{A}(p)>1 / k$ and, for each $u \in \operatorname{supp}(\nu)$,

$$
\varphi\left(u, \tau_{A}\right)= \begin{cases}\{a\} & \text { if } \Phi\left(a, \tau_{A}\right)>0 \text { for some } a \in A \text { with }(u, a) \in \operatorname{supp}(\tau) \\ A_{0}\left(\tau_{A}\right) & \text { otherwise }\end{cases}
$$


where $A_{0}\left(\tau_{A}\right)=\left\{a \in A: \Phi\left(a, \tau_{A}\right)=0\right\}$. In short, the condition $\sum_{p \geq p^{*}\left(\tau_{A}\right)} \tau_{A}(p)>1 / k$ takes care of the continuity problem and the remaining condition takes case of the non-strict problem by treating all actions in $A_{0}\left(\tau_{A}\right)$ as one.

\subsection{Allocation of schools}

Consider, for each $n \in \mathbb{N}$, ns schools, each with $m \in \mathbb{N}$ seats, and $n k$ students, where $s, k \in \mathbb{N}$. Each student chooses one school and, as a result of the profile of choices, he may or may not get a seat in the chosen school. We let $A_{n}=\left\{\frac{j}{n s}: j=1, \ldots, n s\right\}$ represent the set of schools. The set of players are the students: $I_{n}=\{1, \ldots, n k\}$. Each student's action set is $A_{n}$ and, given an action profile $\tilde{a}=\left(\tilde{a}_{1}, \ldots, \tilde{a}_{n k}\right)$, student $i$ 's payoff is

$$
u_{i}\left(\tilde{a}_{i}, \frac{1}{n-1} \sum_{j \in I_{n} \backslash\{i\}} 1_{\tilde{a}_{j}}\right) .
$$

The interpretation of this formalization is analogous to the one in Section 8.1. However, unlike it, here players' action sets change with the number of players and, in fact, its sequence converges to $[0,1]$ in the Hausdorff distance. Thus, we cannot use Theorems 1 and 2 here, but Theorems 4 and 5 do apply to this problem.

\section{Concluding remarks}

The main message of this paper is that non-atomic games are closely related to, and therefore are good idealizations of, large finite-player games. This has been established for the standard framework of large games (where each player's payoff functions depends continuously on his action and on the entire distribution of actions chosen by the other players) using the standard equilibrium concept — Nash equilibrium — in both classes of games.

Our first set of results shows that a well-know property of non-atomic games - existence of pure strategy Nash equilibria - extends generically to large finiteplayer games. The "generic" qualification is unavoidable because the distribution of players' payoff functions in some games can prevent pure strategy Nash equilibria 
to exist in sufficiently large finite-player games. These examples being non-generic means that the distribution of players' payoff functions in any such example can be slightly perturbed to give rise to a game where the existence of pure strategy Nash equilibria in sufficiently large games is guaranteed in a robust way: This property is not lost if the distribution of players' payoff functions of the latter game is further perturbed.

Our second set of results shows that equilibria of non-atomic games are limits of equilibria of large finite games. This result holds for each equilibrium distribution of each non-atomic game, not just for generic ones.

Our results are weaker in the case of games with a general compact metric action space and require the presence of a small amount of mixing in the players' choices. We do not regard this as discouraging: The presence of infinitely many actions close to each other creates difficulties for our approach - players have, in an informal sense, too much choice. Under the differentiability assumptions made in Carmona and Podczeck (2019), this is not a problem because, generically, optimal choices are locally unique; here, in the absence of these assumptions, this problems creates the need for the use of mixed strategies that are precisely concentrated in a set of actions that are close to each other.

We leave for future research the question of whether or not mixed strategies can be completely dispensed with in our generic asymptotic existence result in the general case of a compact metric action universe. In addition, we also leave for future research the question of whether or not our results can be extended to the case of incomplete information as in Kalai (2004).

\section{A Appendix}

\section{A.1 Roadmap}

In this appendix we prove our results. Some additional notation is presented in Section A.2. In Section A.3, several lemmas are stated and proved. 
We start with the details of the topology on the space $\mathcal{U}$ of payoff functions and the characterization of its convergence notion in Lemma 2. Lemmas 3-5 then state several properties of the space $\mathcal{M}$. We then present a lemma, Lemma 6 , stating that each non-atomic game has a sequence of finite-player games converging to it; this is a simple matter when players' payoffs are assumed to be defined on the entire space $A \times M(A)$ but it is more delicate when, as in this paper, this is not assumed.

Section A.3 also includes two lemmas (one for the case when $A$ is finite and another for the case when $A$ is compact) where any equilibrium distributions of any non-atomic games is approximated by equilibrium distributions of non-atomic games having additional robustness properties: The latter are strict equilibrium distributions in the finite case, and symmetric and continuous distributions in the compact case.

The proof of each of the results in the main text is presented in Section A.4.

Throughout this appendix it is assumed that $A$ is a compact metric space unless explicitly stated otherwise.

\section{A.2 Notation}

Given a compact metric space $X$, we use $\xi$ to denote the Hausdorff distance on the space of nonempty compact subsets of $X$. A more precise notation would be $\xi_{X}$; we omit the subscript but will be clear from the context what $X$ is. The definition of $\xi$ is as follows: Given two nonempty compact subsets $C$ and $D$ of $X$,

$$
\xi(C, D)=\max \left\{\sup _{x \in C} d(x, D), \sup _{x \in D} d(x, C)\right\}
$$

where $d(x, D)=\inf _{x^{\prime} \in D} d\left(x, x^{\prime}\right)$ and analogously for $d(x, C)$, and where $d$ denotes a metric on $X$.

Likewise, given a separable metric space $X$, we let $\rho$ denote the Huntingdon's metric on the space $M(X)$ of Borel probability measures on $X$. It is defined as follows: Let $C(X)$ be the set of all real-valued continuous functions on $X$, and let $L=\{h \in C(X):\|h\| \leq 1$ and $h$ is 1 -Lipschitz $\}$. Then $\rho$ is defined by setting $\rho\left(\mu, \mu^{\prime}\right)=\sup \left\{\left|\int h d \mu-\int h d \mu^{\prime}\right|: h \in L\right\}$ for all $\mu, \mu^{\prime} \in M(X)$. Recall that this metric induces the narrow topology. In the sequel, notions of open or closed balls in $M(X)$ 
are always understood as referring to $\rho$. Note that because $\rho$ is induced by a norm on the set of all signed Borel measures on $X$, if $g_{i}$ and $f_{i}, i=1, \ldots, k$, are elements of $M(X)$ with $\rho\left(g_{i}, f_{i}\right) \leq \delta$ for all $i$, then $\rho\left(\frac{1}{k} \sum_{i=1}^{k} g_{i}, \frac{1}{k} \sum_{i=1}^{k} f_{i}\right) \leq \delta$. Note also that by the definition of Huntingdon's metric, if $d\left(x, x^{\prime}\right) \leq \delta$ for $x, x^{\prime} \in X$, where $d$ is the metric of $X$, then $\rho\left(1_{x}, 1_{x^{\prime}}\right) \leq \delta$ for the corresponding Dirac measures $1_{x}$ and $1_{x^{\prime}}$.

\section{A.3 Lemmata}

\section{A.3.1 $\quad$ Space $\mathcal{U}$}

Recall that $\mathcal{U}$ denotes the space of all continuous functions $u: A^{\prime} \times M^{\prime} \rightarrow \mathbb{R}$ where $A^{\prime}$ is a nonempty and closed subset of $A$ and $M^{\prime}$ is a nonempty and closed subset of $M(A)$, and that $\operatorname{dom}(u)$ denotes the domain of $u \in \mathcal{U}$. We identify any $u \in \mathcal{U}$ with its graph: $\operatorname{graph}(u)=\left\{(a, \pi, \alpha) \in A^{\prime} \times M^{\prime} \times \mathbb{R}: \alpha=u(a, \pi)\right\}$. As $\operatorname{graph}(u)$ is a nonempty compact subset of $A \times M(A) \times \mathbb{R}$ for each $u \in \mathcal{U}$, we identify $\mathcal{U}$ with a subset of the space of nonempty and compact subsets of $A \times M(A) \times \mathbb{R}$ which, when endowed with the Hausdorff distance, is a separable metric space. Thus, $\mathcal{U}$ is a separable metric space. We let $d$ denote the metric on $\mathcal{U}$, defined by setting, for each $u, v \in \mathcal{U}, d(u, v)$ to be the Hausdorff distance between $\operatorname{graph}(u)$ and $\operatorname{graph}(v)$.

Lemma 2. Let $u, u_{k} \in \mathcal{U}$ for each $k \in \mathbb{N}$. Then $d\left(u_{k}, u\right) \rightarrow 0$ if and only if $(a)$ $\operatorname{dom}\left(u_{k}\right) \rightarrow \operatorname{dom}(u)$ in the Hausdorff metric topology and $(b) u_{k}\left(x_{k}\right) \rightarrow u(x)$ whenever $x_{k} \rightarrow x, x \in \operatorname{dom}(u)$ and $x_{k} \in \operatorname{dom}\left(u_{k}\right)$ for each $k \in \mathbb{N}$.

Proof. (Necessity) Suppose that $d\left(u_{k}, u\right) \rightarrow 0$. We have that $\xi\left(\operatorname{dom}\left(u_{k}\right), \operatorname{dom}(u)\right) \leq$ $\xi\left(\operatorname{graph}\left(u_{k}\right), \operatorname{graph}(u)\right)$ for each $k$, which established (a). Regarding (b), we have that $\left(x_{k}, u_{k}\left(x_{k}\right)\right) \in \operatorname{graph}\left(u_{k}\right)$ for each $k \in \mathbb{N}$. Since $u_{k} \rightarrow u$, there exists $\left\langle x_{k}^{\prime}\right\rangle$ such that $x_{k}^{\prime} \in \operatorname{dom}(u)$ for each $k \in \mathbb{N},\left|u_{k}\left(x_{k}\right)-u\left(x_{k}^{\prime}\right)\right| \rightarrow 0$ and the distance between $x_{k}^{\prime}$ and $x_{k}$ also goes to zero. Thus, $x_{k}^{\prime} \rightarrow x, u\left(x_{k}^{\prime}\right) \rightarrow u(x)$ (because $u$ is continuous) and $\left|u_{k}\left(x_{k}\right)-u(x)\right| \leq\left|u_{k}\left(x_{k}\right)-u\left(x_{k}^{\prime}\right)\right|+\left|u\left(x_{k}^{\prime}\right)-u(x)\right| \rightarrow 0$. Therefore, $u_{k}\left(x_{k}\right) \rightarrow u(x)$.

(Sufficiency) Suppose that (a) and (b) hold. It then follows immediately that $\operatorname{graph}(u) \subseteq \operatorname{Ligraph}\left(u_{k}\right) .{ }^{13} \operatorname{Suppose}$ that $(x, r) \in \operatorname{Ls} \operatorname{graph}\left(u_{k}\right)$. Then for some se-

\footnotetext{
${ }^{13}$ Here and below, $\operatorname{Ligraph}\left(u_{k}\right)$ is the set of limits of sequences $\left\langle\left(x_{k}, r_{k}\right)\right\rangle$ such that $\left(x_{k}, r_{k}\right) \in$
} 
quence $\left\langle n_{i}\right\rangle_{i \in \mathbb{N}}$ in $\mathbb{N}$ there are points $x_{k_{i}} \in \operatorname{dom}\left(u_{k_{i}}\right), i \in \mathbb{N}$, such that $\left(x_{k_{i}}, u_{k_{i}}\left(x_{k_{i}}\right)\right) \rightarrow$ $(x, r)$. From (a) we see that $x \in \operatorname{dom}(u)$. Again from (a), there is a sequence $\left\langle x_{k}\right\rangle_{k \in \mathbb{N}}$ such that $x_{k} \rightarrow x$ and $x_{k} \in \operatorname{dom}\left(u_{k}\right)$ for each $k$. Define a sequence $\left\langle x_{k}^{\prime}\right\rangle_{k \in \mathbb{N}}$ by setting $x_{k}^{\prime}=x_{k_{i}}$ if $k=k_{i}$ for some $i$, and $x_{k}^{\prime}=x_{k}$ otherwise. Then $x_{k}^{\prime} \in \operatorname{dom}\left(u_{k}\right)$ for each $k$ and $x_{k}^{\prime} \rightarrow x$, so (b) implies that $u_{k}\left(x_{k}^{\prime}\right) \rightarrow u(x)$. In particular, we have $u_{k_{i}}\left(x_{k_{i}}\right) \rightarrow u(x)$ and, therefore, $r=u(x)$. Thus, Ls graph $\left(u_{k}\right) \subseteq \operatorname{graph}(u)$ and it follows that $\operatorname{graph}(u)=\operatorname{Lsgraph}\left(u_{k}\right)=\operatorname{Li} \operatorname{graph}\left(u_{k}\right)$.

Because $\operatorname{dom}(u)$ and $\operatorname{dom}\left(u_{k}\right), k \in \mathbb{N}$, are all included in the compact set $A \times M(A)$, and because the maps $u$ and $u_{k}$ are continuous, (a) and (b) imply, in particular, that the sets $\operatorname{graph}(u)$ and $\operatorname{graph}\left(u_{k}\right), k \in \mathbb{N}$, are commonly included in a compact subset of $A \times M(A) \times \mathbb{R}$, so the fact that $\operatorname{graph}(u)=\operatorname{Ls} \operatorname{graph}\left(u_{k}\right)=\operatorname{Ligraph}\left(u_{k}\right)$ implies that $d\left(u_{k}, u\right)=\xi\left(\operatorname{graph}\left(u_{k}\right), \operatorname{graph}(u)\right) \rightarrow 0$.

\section{A.3.2 Space $\mathcal{M}$}

Lemma 3. Let $\left\langle\nu_{n}\right\rangle_{n \in \mathbb{N}}$ be a sequence in $\mathcal{M}$ and $\nu \in \mathcal{M}$. If $\nu_{n} \rightarrow \nu$ in the narrow topology and, for each $\varepsilon>0$, there exists $N \in \mathbb{N}$ such that $\operatorname{supp}\left(\nu_{n}\right) \subseteq B_{\varepsilon}(\operatorname{supp}(\nu))$ for all $n \geq N$, then $\operatorname{supp}\left(\nu_{n}\right) \rightarrow \operatorname{supp}(\nu)$ in the Hausdorff metric topology.

Proof. Let $\varepsilon>0$. It clearly suffices to show that there is $N \in \mathbb{N}$ such that, for each $n \geq N$ and $u \in \operatorname{supp}(\nu)$, there exists $u^{\prime} \in \operatorname{supp}\left(\nu_{n}\right)$ such that $d\left(u, u^{\prime}\right)<2 \varepsilon$.

Using the compactness of $\operatorname{supp}(\nu)$, let $u_{1}, \ldots, u_{J} \in \operatorname{supp}(\nu)$ be such that $\operatorname{supp}(\nu) \subseteq$ $\bigcup_{j=1}^{J} B_{\varepsilon}\left(u_{j}\right)$. For each $j$, we have that $\nu\left(B_{\varepsilon}\left(u_{j}\right)\right)>0$ since $u_{j} \in \operatorname{supp}(\nu)$. As $\nu_{n} \rightarrow \nu$ in the narrow topology, $\nu\left(B_{\varepsilon}\left(u_{j}\right)\right) \leq \liminf _{n} \nu_{n}\left(B_{\varepsilon}\left(u_{j}\right)\right)$; thus, there is $N \in \mathbb{N}$ such that, for each $n \geq N$ and $j=1, \ldots, J, \nu_{n}\left(B_{\varepsilon}\left(u_{j}\right)\right)>0$. This then implies that $B_{\varepsilon}\left(u_{j}\right) \cap \operatorname{supp}\left(\nu_{n}\right) \neq \emptyset$ for each $n \geq N$ and $j=1, \ldots, J$. Thus, for each $n \geq N$ and $u \in \operatorname{supp}(\nu)$, there exists $u^{\prime} \in \operatorname{supp}\left(\nu_{n}\right)$ such that $d\left(u, u^{\prime}\right)<2 \varepsilon$.

Lemma 4. If $\nu_{k} \rightarrow \nu \in \mathcal{M}$ and $\left\langle u_{k}\right\rangle_{k \in \mathbb{N}}$ is a sequence in $\mathcal{U}$ with $u_{k} \in \operatorname{supp}\left(\nu_{k}\right)$ for each $k$, then there is a subsequence $\left\langle u_{k_{n}}\right\rangle_{n \in \mathbb{N}}$ of $\left\langle u_{k}\right\rangle_{k \in \mathbb{N}}$ and $u \in \operatorname{supp}(\nu)$ such that $u_{k_{n}} \rightarrow u$.

$\operatorname{graph}\left(u_{k}\right)$ for all $k$, and $\operatorname{Lsgraph}\left(u_{k}\right)$ is the set of cluster points of such sequences. 
Proof. By the choice of the topology on $\mathcal{M}, \nu_{k} \rightarrow \nu \in \mathcal{M}$ implies that $\operatorname{supp}\left(\nu_{k}\right) \rightarrow$ $\operatorname{supp}(\nu)$ in the Hausdorff metric topology. Thus if $\left\langle u_{k}\right\rangle$ is a sequence in $\mathcal{U}$ with $u_{k} \in$ $\operatorname{supp}\left(\nu_{k}\right)$ for each $k$, there is a sequence $\left\langle u_{k}^{\prime}\right\rangle$ in $\operatorname{supp}(\nu)$ such that $d\left(u_{k}, u_{k}^{\prime}\right) \rightarrow 0$. Now since $\operatorname{supp}(\nu)$ is compact, $\left\langle u_{k}^{\prime}\right\rangle$ has a convergent subsequence, say with limit $u \in \operatorname{supp}(\nu)$. Evidently the corresponding subsequence of $\left\langle u_{k}\right\rangle$ converges to $u$, too.

Recall that the domain of $u \in \mathcal{U}$ is denoted by $A_{u} \times M_{u}$.

Lemma 5. The following holds:

(a) For every $\nu \in \mathcal{M}, E(\nu)$ is a compact convex subset of $M(A)$ and $E(\nu)=$ $\int_{\mathcal{U}} M\left(A_{u}\right) \mathrm{d} \nu(u)$.

(b) If $\nu_{n} \rightarrow \nu$ in $\mathcal{M}$, then $E\left(\nu_{n}\right) \rightarrow E(\nu)$ for the Hausdorff metric on the set of all non-empty compact subsets of $M(A)$.

Proof. Clearly $E(\nu)$ is compact and convex for each $\nu \in \mathcal{M}$. For the other claims, consider the correspondences $\Lambda: \mathcal{U} \rightarrow 2^{A}$ and $\theta: \mathcal{U} \rightarrow 2^{M(A)}$ defined by setting

$$
\Lambda(u)=A_{u} \text { and } \theta(u)=M\left(A_{u}\right)
$$

for each $u \in \mathcal{U}$. Then $\Lambda$ is a continuous correspondence with non-empty compact values; in particular, $\operatorname{graph}(\Lambda)$ is closed.

We first claim that $E(\nu)=\int \theta(u) d \nu(u)$ for each $\nu \in \mathcal{M}$. To see this, let $\pi \in E(\nu)$ and $\tau \in M(\mathcal{U} \times A)$ be such that $\tau_{A}=\pi, \tau_{\mathcal{U}}=\nu$ and $\tau(\operatorname{graph}(\Lambda))=1$. There exists a measurable function $f: \mathcal{U} \rightarrow M(A)$ such that

$$
\tau(B \times C)=\int_{B} f(u)(C) \mathrm{d} \nu(u) \text { for each Borel } B \subseteq \mathcal{U} \text { and Borel } C \subseteq A \text {. }
$$

In particular, $1=\tau(\operatorname{graph}(\Lambda))=\int_{\mathcal{U}} f(u)\left(A_{u}\right) \mathrm{d} \nu(u)$; hence, changing $f$ in a $\nu$ negligible set if necessary, we may assume that $f(u) \in M\left(A_{u}\right)$ for each $u \in \mathcal{U}$. Thus, $\pi=\tau_{A}=\int_{\mathcal{U}} f \mathrm{~d} \nu \in \int_{\mathcal{U}} \theta \mathrm{d} \nu$.

Conversely, let $\pi \in \int_{\mathcal{U}} \theta \mathrm{d} \nu$ and let $f: \mathcal{U} \rightarrow M(A)$ be a measurable selection of $\theta$ such that $\pi=\int_{\mathcal{U}} f \mathrm{~d} \nu$. Define $\tau \in M(\mathcal{U} \times A)$ by (4). Then $\tau_{A}=\int_{\mathcal{U}} f \mathrm{~d} \nu=\pi, \tau_{\mathcal{U}}=\nu$ and $\tau(\operatorname{graph}(\Lambda))=\int_{\mathcal{U}} f(u)\left(A_{u}\right) \mathrm{d} \nu(u)=1$. Thus, $\pi \in E(\nu)$. 
As for part (b) of the lemma, first note that $\theta$ is continuous and has non-empty compact convex values. To see the continuity of $\theta$ (the remaining properties are clear), take $u_{k} \rightarrow u$, which implies that $A_{u_{k}} \rightarrow A_{u}$. If $\pi \in M\left(A_{u}\right)$, let $\pi_{k}^{\prime} \rightarrow \pi$ be such that $\pi_{k}^{\prime} \in M\left(A_{u}\right)$ has finite support for each $k$. For each $k \in \mathbb{N}$ and $a^{\prime} \in \operatorname{supp}\left(\pi_{k}^{\prime}\right)$, there exists $a \in A_{u_{k}}$ such that $d\left(a, a^{\prime}\right)<\xi\left(A_{u}, A_{u_{k}}\right)+1 / k$; set $\pi_{k}(\{a\})=\pi_{k}^{\prime}\left(\left\{a^{\prime}\right\}\right)$. Then $\pi_{k} \in M\left(A_{u_{k}}\right)$ and $\rho\left(\pi_{k}, \pi_{k}^{\prime}\right) \leq \xi\left(A_{u}, A_{u_{k}}\right)+1 / k$; in particular, $\pi_{k} \rightarrow \pi$. Conversely, if $\pi=\lim \pi_{k_{j}}$ with $\pi_{k_{j}} \in M\left(A_{u_{k_{j}}}\right)$ for each $j \in \mathbb{N}$, then $\pi\left(B_{\varepsilon}\left(A_{u}\right)\right) \geq \pi\left(\bar{B}_{\varepsilon / 2}\left(A_{u}\right)\right) \geq$ $\limsup \sup _{k_{j}}\left(\bar{B}_{\varepsilon / 2}\left(A_{u}\right)\right)=1$ for each $\varepsilon>0$ since $A_{u_{k_{j}}} \subseteq \bar{B}_{\varepsilon / 2}\left(A_{u}\right)$ for all $j$ sufficiently large; hence, $\pi\left(A_{u}\right)=\pi\left(\bigcap_{l=1}^{\infty} B_{1 / l}\left(A_{u}\right)\right)=1$ and $\pi \in M\left(A_{u}\right)$.

Note also that $M(A)$ is a subset of a Hausdorff locally convex space and let $E^{\prime}$ denote its dual. For each $p \in E^{\prime}$, the map $u \mapsto \max _{\pi \in \theta(u)} p(\pi): \mathcal{U} \rightarrow \mathbb{R}$ is continuous. Moreover, this map is bounded because the values of $\theta$ are included in the compact set $M(A)$. Hence, for each $p \in E^{\prime}$, the map $\nu \mapsto \int_{\mathcal{U}} \max _{\pi \in \theta(u)} p(\pi) d \nu(u): \mathcal{M} \rightarrow \mathbb{R}$ is continuous.

We have

$$
\max _{\pi \in \int_{\mathcal{U}} \theta(u) d \nu(u)} p(\pi)=\int_{\mathcal{U}} \max _{\pi \in \theta(u)} p(\pi) d \nu(u) .
$$

Indeed, let $f$ be a measurable selection of $\theta$ such that

$$
\max _{\pi \in \int_{\mathcal{U}} \theta(u) d \nu(u)} p(\pi)=p\left(\int_{\mathcal{U}} f(u) d \nu(u)\right)
$$

and $h$ be a measurable selection of $\theta$ such that $p(h(u))=\max _{\pi \in \theta(u)} p(\pi)$ for each $u \in \mathcal{U}$. Then

$$
\begin{aligned}
& \max _{\pi \in \int_{\mathcal{U}} \theta(u) d \nu(u)} p(\pi)=p\left(\int_{\mathcal{U}} f(u) d \nu(u)\right)=\int_{\mathcal{U}} p(f(u)) d \nu(u) \leq \int_{\mathcal{U}} \max _{\pi \in \theta(u)} p(\pi) d \nu(u) \\
& =\int_{\mathcal{U}} p(h(u)) d \nu(u)=p\left(\int_{\mathcal{U}} h(u) d \nu(u)\right) \leq \max _{\pi \in \int_{\mathcal{U}} \theta(u) d \nu(u)} p(\pi),
\end{aligned}
$$

from which (5) follows.

It follows by (5) that, for each $p \in E^{\prime}$, the map $\nu \mapsto \max _{\pi \in \int_{\mathcal{U}} \theta(u) d \nu(u)} p(\pi): \mathcal{M} \rightarrow \mathbb{R}$ is continuous. Because $\int_{\mathcal{U}} \theta(u) d \nu(u)$ is a non-empty convex and compact subset of the compact set $M(A)$ for each $\nu \in \mathcal{M}$, it follows from this that the map $\nu \mapsto$ 
$\int_{\mathcal{U}} \theta(u) d \nu(u)$ is continuous for the Hausdorff metric on the set of all non-empty compact subsets of $M(A)$ (see Castaing and Valadier (1977, Corollary II-22, p. 53)). Thus we get (b), again by the equality established above.

\section{A.3.3 Approximation of games}

Lemma 6. For each $\mu \in \mathcal{G}$, there exists a sequence $\left\langle\left(I_{n}, G_{n}\right)\right\rangle_{n \in \mathbb{N}}$ of finite-player games such that $\nu_{\left(I_{n}, G_{n}\right)} \rightarrow \mu$ in $\mathcal{M}$ and $\#\left(I_{n}\right) \rightarrow \infty$.

Proof. Let $\left\langle\mu_{n}\right\rangle_{n \in \mathbb{N}}$ be such that $\mu_{n} \rightarrow \mu$ narrowly and, for each $n \in \mathbb{N}, \operatorname{supp}\left(\mu_{n}\right)$ is a finite subset of $\operatorname{supp}(\mu)$ and $\mu_{n}(\{u\}) \in \mathbb{Q}$ for each $u \in \operatorname{supp}\left(\mu_{n}\right)$. Note that Lemma 3 implies that $\mu_{n} \rightarrow \mu$ in the topology of $\mathcal{M}$.

Fix $n \in \mathbb{N}$ and let $k_{n} \in \mathbb{N}$ be such that, for each $u \in \operatorname{supp}\left(\mu_{n}\right), \mu_{n}(\{u\})=p_{u} / k_{n}$ for some $p_{u} \in \mathbb{N}$. For each $m \in \mathbb{N}$, let $M_{m k_{n} \mu_{n}(u)}\left(A_{u}\right)$ be the set of $\pi \in M\left(A_{u}\right)$ such that $\pi$ has finite support and, for each $a \in \operatorname{supp}(\pi), \pi(\{a\})=l /\left(m k_{n} \mu_{n}(\{u\})\right)$ for some $0 \leq l \leq m k_{n} \mu_{n}(\{u\})$. Define $M_{m k_{n} \mu_{n}(u)-1}\left(A_{u}\right)$ analogously with $m k_{n} \mu_{n}(u)-1$ in place of $m k_{n} \mu_{n}(u)$. Pick $m_{n} \in \mathbb{N}$ such that $m_{n} k_{n} \geq n, \xi\left(M_{m_{n} k_{n} \mu_{n}(u)}\left(A_{u}\right), M\left(A_{u}\right)\right)<1 / n$ and $\xi\left(M_{m_{n} k_{n} \mu_{n}(u)-1}\left(A_{u}\right), M\left(A_{u}\right)\right)<1 / n$ for each $u \in \operatorname{supp}\left(\mu_{n}\right)$.

To see that such $m_{n}$ exists, let $\pi \in M\left(A_{u}\right)$ and pick $\pi^{\prime} \in M\left(A_{u}\right)$ with finite support such that $\rho\left(\pi, \pi^{\prime}\right)<(2 n)^{-1}$. Write $\left\{a_{1}, \ldots, a_{L}\right\}=\operatorname{supp}\left(\pi^{\prime}\right)$ and $\lambda_{l}=\pi^{\prime}\left(\left\{a_{l}\right\}\right)$ for $l=1, \ldots, L$. Pick $m_{n}$ such that $m_{n} k_{n} \mu_{n}(u)>2 n L$ for each $u \in \operatorname{supp}\left(\mu_{n}\right)$. Moreover, let $j_{1}$ be the largest integer such that $j_{1}\left(m_{n} k_{n} \mu_{n}(u)\right)^{-1} \leq \lambda_{1}$; for $1<l<L$, assuming that $j_{1}, \ldots, j_{l-1}$ have been chosen, let $j_{l}$ be the largest integer such that $\left(j_{1}+\cdots+\right.$ $\left.j_{l}\right)\left(m_{n} k_{n} \mu_{n}(u)\right)^{-1} \leq \lambda_{1}+\cdots+\lambda_{l}$. Finally, let $j_{L}$ be such that $\sum_{l=1}^{L} j_{l}=m_{n} k_{n} \mu_{n}(u)$. It is then easy to show that $\left|j_{l}\left(m_{n} k_{n} \mu_{n}(u)\right)^{-1}-\lambda_{l}\right| \leq\left(m_{n} k_{n} \mu_{n}(u)\right)^{-1}<(2 n L)^{-1}$ for each $l=1, \ldots, L$. Thus, $\tilde{\pi}$ defined by $\tilde{\pi}\left(\left\{a_{l}\right\}\right)=j_{l}\left(m_{n} k_{n} \mu_{n}(u)\right)^{-1}$ for $l=1, \ldots, L$ is such that $\tilde{\pi} \in M_{m_{n} k_{n} \mu_{n}(u)}\left(A_{u}\right)$ and $\rho\left(\pi^{\prime}, \tilde{\pi}\right)<(2 n)^{-1}$. Do a similar construction with $m_{n} k_{n} \mu_{n}(u)-1$ in place of $m_{n} k_{n} \mu_{n}(u)$.

For each $n \in \mathbb{N}$, define $\left(I_{n}, \hat{G}_{n}\right)$ such that $\#\left(I_{n}\right)=m_{n} k_{n} \geq n$ and $\nu_{\left(I_{n}, \hat{G}_{n}\right)}=\mu_{n}$ (i.e. $m_{n} k_{n} \mu_{n}(u)$ players $i \in I_{n}$ have $\hat{G}_{n}(i)=u$ for each $\left.u \in \operatorname{supp}\left(\mu_{n}\right)\right) ;\left(I_{n}, \hat{G}_{n}\right)$ is not necessarily a game because $\operatorname{dom}\left(\hat{G}_{n}(i)\right)$ may fail to be $A_{i} \times E_{i}$. For this reason, we modify the function $\hat{G}_{n}$ as follows. 
Note that $\operatorname{supp}(\mu)$ is bounded and equicontinuous by Lemma 1. Because $\operatorname{supp}(\mu)$ is bounded, we may assume that each $u \in \operatorname{supp}(\mu)$ takes values in $[1,2]$; then, for each $u \in \operatorname{supp}(\mu)$, define $\tilde{u}: A_{u} \times M(A) \rightarrow[1,2]$ by setting

$$
\tilde{u}(a, \pi)= \begin{cases}u(a, \pi) & \text { if } \pi \in E(\mu), \\ \inf _{\pi^{\prime} \in E(\mu) \frac{u\left(a, \pi^{\prime}\right) \rho\left(\pi, \pi^{\prime}\right)}{d(\pi, E(\mu))}} & \text { otherwise. }\end{cases}
$$

Because $\operatorname{supp}(\mu)$ is equicontinuous, the proof of the theorem in Mandelkern (1990) shows that $U=\{\tilde{u}: u \in \operatorname{supp}(\mu)\}$ is also bounded and equicontinuous.

We now define $G_{n}$ as follows. Let $i \in I_{n}$ be given. Denote $\hat{G}_{n}(i)$ by $v_{i}$, $\operatorname{dom}\left(v_{i}\right)$ by $A_{i} \times E(\mu)\left(\right.$ recall that $\left.v_{i} \in \operatorname{supp}(\mu)\right)$ and denote by $E_{n, i}$ the set in (1) (note that $n$ in that formula needs to be replaced with \# $\left.\left(I_{n}\right)\right)$. Define $G_{n}(i)$ be the restriction of $\tilde{v}_{i}$ to $A_{i} \times E_{n, i}$.

We have that $E\left(\mu_{n}\right) \rightarrow E(\mu)$ by Lemma 5 . We claim that $\xi\left(E\left(\mu_{n}\right), E_{n, i}\right) \leq 3 /(n-$ 1) for each $n \in \mathbb{N}$ and $i \in I_{n}$. Indeed, letting $l_{n}=\#\left(I_{n}\right)=m_{n} k_{n}$,

$$
\begin{aligned}
& E\left(\mu_{n}\right)=\sum_{u \in \operatorname{supp}\left(\mu_{n}\right)} \mu_{n}(u) M\left(A_{u}\right) \text { and } \\
& E_{n, i}=\sum_{u \in \operatorname{supp}\left(\mu_{n}\right): u \neq u_{i}} \frac{l_{n} \mu_{n}(u)}{l_{n}-1} M_{l_{n} \mu_{n}(u)}\left(A_{u}\right)+\frac{l_{n} \mu_{n}\left(u_{i}\right)-1}{l_{n}-1} M_{l_{n} \mu_{n}(u)-1}\left(A_{u_{i}}\right) .
\end{aligned}
$$

Hence, for any $\pi \in E\left(\mu_{n}\right)$, we have that $\pi=\sum_{u \in \operatorname{supp}\left(\mu_{n}\right)} \mu_{n}(u) \pi_{u}$ with $\pi_{u} \in M\left(A_{u}\right)$ for each $u \in \operatorname{supp}\left(\mu_{n}\right)$. Let $\pi_{u_{i}}^{\prime} \in M_{l_{n} \mu_{n}\left(u_{i}\right)-1}\left(A_{u_{i}}\right)$ be such that $\rho\left(\pi_{u_{i}}, \pi_{u_{i}}^{\prime}\right)<1 / n$ and, for each $u \in \operatorname{supp}\left(\mu_{n}\right)$ with $u \neq u_{i}$, let $\pi_{u}^{\prime} \in M_{l_{n} \mu_{n}(u)}\left(A_{u}\right)$ be such that $\rho\left(\pi_{u}, \pi_{u}^{\prime}\right)<1 / n$. Write $\mu_{n}^{\prime}\left(u_{i}\right)=\frac{l_{n} \mu_{n}\left(u_{i}\right)-1}{l_{n}-1}$ and $\mu_{n}^{\prime}(u)=\frac{l_{n} \mu_{n}(u)}{l_{n}-1}$ for each $u \in \operatorname{supp}\left(\mu_{n}\right)$ with $u \neq u_{i}$. Define $\pi^{\prime}=\sum_{u \in \operatorname{supp}\left(\mu_{n}\right)} \mu_{n}(u) \pi_{u}^{\prime}$ and $\tilde{\pi}=\sum_{u \in \operatorname{supp}\left(\mu_{n}\right)} \mu_{n}^{\prime}(u) \pi_{u}^{\prime}$. We have that $\tilde{\pi} \in E_{n, i}$, $\rho\left(\pi, \pi^{\prime}\right) \leq \frac{1}{n}$ and

$$
\begin{aligned}
\rho\left(\pi^{\prime}, \tilde{\pi}\right) & \leq \sum_{u \in \operatorname{supp}\left(\mu_{n}\right)}\left|\mu_{n}^{\prime}(u)-\mu_{n}(u)\right|=\frac{1}{l_{n}-1}\left(\sum_{u \in \operatorname{supp}\left(\mu_{n}\right): u \neq u_{i}} \mu_{n}(u)+1-\mu_{n}\left(u_{i}\right)\right) \\
& =\frac{2\left(1-\mu_{n}\left(u_{i}\right)\right)}{l_{n}-1} \leq \frac{2}{l_{n}-1} \leq \frac{2}{n-1} .
\end{aligned}
$$

The argument to show that, for each element of $E_{n, i}$, there is an element of $E\left(\mu_{n}\right)$ whose distance is no greater than $3 /(n-1)$ is analogous, hence the conclusion follows. 
For each $n \in \mathbb{N}$, let $\theta_{n}=\xi\left(E\left(\mu_{n}\right), E(\mu)\right)+3 / n$ and $\omega\left(\theta_{n}\right)=\sup _{u \in U} \sup \{\mid u(a, \pi)-$ $\left.u\left(a, \pi^{\prime}\right) \mid: a \in A_{u}, \pi, \pi^{\prime} \in M(A),\left\|\pi-\pi^{\prime}\right\| \leq \theta_{n}\right\}$. We have that $\xi\left(E(\mu), E_{n, i}\right)<\theta_{n}$ for each $n \in \mathbb{N}$ and $i \in I_{n}, \theta_{n} \rightarrow 0$ and $\omega\left(\theta_{n}\right) \rightarrow 0$ (because $U$ is equicontinuous). Hence, for each $n \in \mathbb{N}$ and $i \in I_{n}, d\left(u_{i}, \hat{u}_{i}\right) \leq \max \left\{\theta_{n}, \omega\left(\theta_{n}\right)\right\}$. Indeed, given $\left(a, \pi, u_{i}(a, \pi)\right) \in$ $\operatorname{graph}\left(u_{i}\right)$, there exists $\pi^{\prime} \in E(\nu)$ such that $\left\|\pi-\pi^{\prime}\right\|<\theta_{n}$ and, therefore,

$$
\left|u_{i}(a, \pi)-\hat{u}_{i}\left(a, \pi^{\prime}\right)\right|=\left|\tilde{u}_{i}(a, \pi)-\tilde{u}_{i}\left(a, \pi^{\prime}\right)\right| \leq \omega\left(\theta_{n}\right) .
$$

The argument to show that, for each element of $\operatorname{graph}\left(\hat{u}_{i}\right)$, there is an element of $\operatorname{graph}\left(u_{i}\right)$ whose distance is no greater than $\max \left\{\theta_{n}, \omega\left(\theta_{n}\right)\right\}$ is analogous, hence the conclusion follows.

It then follows that both $\rho\left(\mu_{n}, \nu_{\left(I_{n}, G_{n}\right)}\right)$ and $\xi\left(\operatorname{supp}\left(\mu_{n}\right), \operatorname{supp}\left(\nu_{\left(I_{n}, G_{n}\right)}\right)\right)$ are no greater than $\max \left\{\theta_{n}, \omega\left(\theta_{n}\right)\right\}$. Since $\mu_{n} \rightarrow \mu$ in the topology of $\mathcal{M}$, it then follows that $\nu_{\left(I_{n}, G_{n}\right)} \rightarrow \mu$ in the topology of $\mathcal{M}$ as well.

\section{A.3.4 Approximation of equilibrium distributions}

\section{Case 1: $A$ finite}

Given $\nu \in \mathcal{G}$ and an equilibrium distribution $\tau$ of $\nu$, we say that $\tau$ is strict if $\#\left(\varphi\left(u, \tau_{A}\right)\right)=1$ for each $u \in \operatorname{supp}(\nu)$. Let $\mathcal{G}^{*}$ be the set of $\nu \in \mathcal{G}$ that have a strict equilibrium distribution.

Lemma 7. Suppose that $A$ is finite. If $\nu \in \mathcal{G}$ and $\tau$ is an equilibrium distribution of $\nu$, then there exists $\left\langle\left(\nu_{n}, \tau_{n}\right)\right\rangle_{n \in \mathbb{N}}$ such that $\nu_{n} \in \mathcal{G}^{*}$ and $\tau_{n}$ is a strict equilibrium distribution of $\nu_{n}$ for all $n \in \mathbb{N}, \nu_{n} \rightarrow \nu$ in the topology of $\mathcal{G}$ and $\tau_{n} \rightarrow \tau$ narrowly.

Proof. Fix $\nu \in \mathcal{G}$ and let $\tau$ be an equilibrium distribution for $\nu$. Note that $\operatorname{supp}(\tau)$ is compact. Given $n \in \mathbb{N}$ and $(u, a) \in \mathcal{U} \times A$, let $u_{u, a, n} \in \mathcal{U}$ by defined by setting $\operatorname{dom}\left(u_{u, a, n}\right)=\operatorname{dom}(u)$ and, for each $\left(a^{\prime}, \pi\right) \in A_{u} \times M_{u}$,

$$
u_{u, a, n}\left(a^{\prime}, \pi\right)= \begin{cases}\left(1-\frac{1}{n+1}\right) u\left(a^{\prime}, \pi\right)+\frac{1}{n+1} & \text { if } a^{\prime}=a \\ \left(1-\frac{1}{n+1}\right) u\left(a^{\prime}, \pi\right) & \text { otherwise. }\end{cases}
$$

Define $\kappa_{n}: \mathcal{U} \times A \rightarrow \mathcal{U} \times A$ by setting $\kappa_{n}(u, a)=\left(u_{u, a, n}, a\right)$ for each $(u, a) \in \mathcal{U} \times A$. Observe that $\kappa_{n}$ is continuous for each $n$ (because $A$ is finite and $d\left(u_{u, a, n}, u_{v, a, n}\right) \leq$ 
$d(u, v)$ for each $u, v \in \mathcal{U}, a \in A$ and $n \in \mathbb{N})$ and that $\kappa_{n} \rightarrow i d_{\mathcal{U} \times A}$ uniformly as $n \rightarrow \infty$, writing $i d_{\mathcal{U} \times A}$ for the identity on $\mathcal{U} \times A$ (because $d\left(u_{u, a, n}, u\right) \leq 1 /(n+1)$ for each $u \in \mathcal{U}, a \in A$ and $n \in \mathbb{N}$ ).

Let $\tau_{n}=\tau \circ \kappa_{n}^{-1}$ and $\nu_{n}$ the marginal measure of $\tau_{n}$ on $\mathcal{U}$. Because $\operatorname{proj}_{A} \circ \kappa_{n}=$ $\operatorname{proj}_{A}$ for each $n$, we have $\tau_{n, A}=\tau_{A}$ for each $n$. Of course, $\operatorname{supp}(\tau) \subseteq \kappa_{n}^{-1}\left(\kappa_{n}(\operatorname{supp}(\tau))\right)$ for each $n$, hence

$$
\tau(\operatorname{supp}(\tau))=1=\tau\left(\kappa_{n}^{-1}\left(\kappa_{n}(\operatorname{supp}(\tau))\right)\right)=\tau_{n}\left(\kappa_{n}(\operatorname{supp}(\tau))\right) .
$$

Consequently, $\operatorname{supp}\left(\tau_{n}\right) \subseteq \kappa_{n}(\operatorname{supp}(\tau))$, because $\operatorname{supp}(\tau)$ and, hence, $\kappa_{n}(\operatorname{supp}(\tau))$ are

compact. Thus, if $\left(u^{\prime}, a^{\prime}\right) \in \operatorname{supp}\left(\tau_{n}\right)$, then for some $(u, a) \in \operatorname{supp}(\tau), u^{\prime}=u_{u, a, n}$ and $a^{\prime}=a$; but if $(u, a) \in \operatorname{supp}(\tau)$, then $a \in \varphi\left(u, \tau_{A}\right)$ and $\{a\}=\varphi\left(u_{u, a, n}, \tau_{A}\right)$ by the choice of $u_{u, a, n}$, i.e., $\left\{a^{\prime}\right\}=\varphi\left(u^{\prime}, \tau_{A}\right)=\varphi\left(u^{\prime}, \tau_{n, A}\right)$ because $\tau_{A}=\tau_{n, A}$. Moreover, we have that $\nu_{n} \in \mathcal{G}$ since $\nu \in \mathcal{G}$ and, thus, $M_{u}=E(\nu)=E\left(\nu_{n}\right)$ for each $u \in \operatorname{supp}\left(\nu_{n}\right)$. It follows that, for each $n, \tau_{n}$ is a strict equilibrium distribution for $\nu_{n}$ and that $\nu_{n} \in \mathcal{G}^{*}$.

Finally, note that the fact that $\kappa_{n} \rightarrow i d_{\mathcal{U} \times A}$ uniformly as $n \rightarrow \infty$ implies that $\tau_{n} \rightarrow \tau$ narrowly. In particular, $\nu_{n} \rightarrow \nu$ narrowly. $\operatorname{As} \operatorname{supp}\left(\tau_{n}\right) \subseteq \kappa_{n}(\operatorname{supp}(\tau))$ and $\lim _{n} \sup _{(u, a) \in \mathcal{U} \times A} d\left(u, u_{u, a, n}\right)=0$, it follows that, for each $\varepsilon>0, \operatorname{supp}\left(\nu_{n}\right) \subseteq$ $B_{\varepsilon}(\operatorname{supp}(\nu))$ for all $n$ sufficiently large. Thus, Lemma 3 implies that $\operatorname{supp}\left(\nu_{n}\right) \rightarrow$ $\operatorname{supp}(\nu)$. Consequently, $\nu_{n} \rightarrow \nu$ in the topology of $\mathcal{G}$.

\section{Case 2: $A$ general compact metric space}

Fix $\varepsilon>0$ and $\nu \in \mathcal{G}$. We say that $\tau \in \mathcal{S}_{\varepsilon}(\nu)$ if there is an open set $V \subseteq \mathcal{U}$, with $\operatorname{supp}(\nu) \subseteq V$, numbers $\eta$ and $\delta$, with $0<\eta<\delta \leq \varepsilon / 2$, and a continuous function $s: V \rightarrow A$, with $A_{u} \cap \bar{B}_{\eta}(s(u)) \neq \emptyset$ for each $u \in V$, such that $\tau=\nu \circ\left(i d_{\mathcal{U}}, s\right)^{-1}$ and $u\left(a^{\prime}, \pi\right)>u(a, \pi)$ for all $u \in \operatorname{supp}(\nu), \pi \in \bar{B}_{\delta}\left(\nu \circ s^{-1}\right) \cap E(\nu) a^{\prime} \in A_{u} \cap \bar{B}_{\eta}(s(u))$ and $a \in A_{u} \backslash B_{\delta}(s(u))$. Let $\mathcal{G}_{\varepsilon}^{*}$ be the set of $\nu \in \mathcal{G}$ such that $\mathcal{S}_{\varepsilon}(\nu) \neq \emptyset$; thus, $\mathcal{G}_{\varepsilon}^{*}$ is the set of $\nu \in \mathcal{G}$ such that there exists $V, \eta, \delta$ and $s$ as above.

Lemma 8. If $\nu \in \mathcal{G}$ and $\tau$ is an equilibrium distribution of $\nu$, then there exists $\left\langle\left(\varepsilon_{k}, \nu_{k}, \tau_{k}\right)\right\rangle_{k \in \mathbb{N}}$ such that $\nu_{k} \in \mathcal{G}_{\varepsilon_{k}}^{*}$ and $\tau_{k} \in \mathcal{S}_{\varepsilon_{k}}\left(\nu_{k}\right)$ for all $k \in \mathbb{N}, \varepsilon_{k} \rightarrow 0, \nu_{k} \rightarrow \nu$ in the topology of $\mathcal{G}$ and $\tau_{k} \rightarrow \tau$ narrowly. Furthermore, for each $k \in \mathbb{N}, \nu_{k}$ can be chosen such that $d\left(a, a^{\prime}\right)>\varepsilon_{k}$ for all $a, a^{\prime} \in A_{u}$ and $u \in \operatorname{supp}\left(\nu_{k}\right)$. 
Proof. Fix $\nu \in \mathcal{G}$ and let $\tau$ be an equilibrium distribution for $\nu$. Note that $\operatorname{supp}(\tau)$ is compact. Let $\left\langle\hat{\tau}_{k}\right\rangle$ be a sequence of probability measures on $\mathcal{U} \times A$ with $\hat{\tau}_{k} \rightarrow \tau$ narrowly such that each $\hat{\tau}_{k}$ has a finite support included in $\operatorname{supp}(\tau)$. Write $\hat{\nu}_{k}$ for the marginal measure of $\hat{\tau}_{k}$ on $\mathcal{U}$ and note that $\hat{\nu}_{k} \rightarrow \nu$ narrowly. Since $\operatorname{supp}\left(\hat{\tau}_{k}\right) \subseteq \operatorname{supp}(\tau)$ for all $k$, it follows that $\operatorname{supp}\left(\hat{\nu}_{k}\right) \subseteq \operatorname{supp}(\nu)$ for all $k$. Hence, as $\hat{\nu}_{k} \rightarrow \nu$ narrowly, we also have $\operatorname{supp}\left(\hat{\nu}_{k}\right) \rightarrow \operatorname{supp}(\nu)$ in the Hausdorff metric topology by Lemma 3. Thus $\hat{\nu}_{k} \rightarrow \nu$ in the topology of $\mathcal{G}$.

Note that $\operatorname{supp}(\mu)$ is bounded and equicontinuous by Lemma 1. Because $\operatorname{supp}(\mu)$ is bounded, we may assume that each $u \in \operatorname{supp}(\mu)$ takes values in $[1,2]$; then, for each $u \in \operatorname{supp}(\mu)$, define $\tilde{u}: A_{u} \times M(A) \rightarrow[1,2]$ by setting

$$
\tilde{u}(a, \pi)= \begin{cases}u(a, \pi) & \text { if } \pi \in E(\mu), \\ \inf _{\pi^{\prime} \in E(\mu) \frac{u\left(a, \pi^{\prime}\right) \rho\left(\pi, \pi^{\prime}\right)}{d(\pi, E(\mu))}} & \text { otherwise. }\end{cases}
$$

Because $\operatorname{supp}(\mu)$ is equicontinuous, the proof of the theorem in Mandelkern (1990) shows that $U=\{\tilde{u}: u \in \operatorname{supp}(\mu)\}$ is also bounded and equicontinuous.

Write $\hat{\nu}_{k}=\sum_{i=1}^{i_{k}^{\prime}} \alpha_{i}^{\prime} 1_{\hat{u}_{i}}$ and $A_{i}$ for the projection of $\operatorname{dom}\left(\hat{u}_{i}\right)$ in $A$. Then define $u_{i}$ to be the restriction of $\tilde{\hat{u}}_{i}$ to $A_{i} \times E\left(\hat{\nu}_{k}\right)$ and let $\nu_{k}^{\prime}=\sum_{i=1}^{i_{k}^{\prime}} \alpha_{i}^{\prime} 1_{u_{i}}$. Noting that $E\left(\hat{\nu}_{k}\right)=E\left(\nu_{k}^{\prime}\right)=\sum_{i=1}^{i_{k}^{\prime}} \alpha_{i}^{\prime} M\left(A_{i}\right)$, we have that $\nu_{k}^{\prime} \in \mathcal{G}$.

We have that $E\left(\hat{\nu}_{k}\right) \rightarrow E(\nu)$ by Lemma 5 and, thus, $E\left(\nu_{k}^{\prime}\right) \rightarrow E(\nu)$. Arguing as in the proof of Lemma 6 , we have that $\nu_{k}^{\prime} \rightarrow \nu$ in the topology of $\mathcal{G}$.

Moreover, for each $k \in \mathbb{N}$, write $\hat{\tau}_{k}=\sum_{i=1}^{i_{k}} \alpha_{i} 1_{\left(\hat{u}_{i}, a_{i}\right)}$ and let $\tau_{k}^{\prime}=\sum_{i=1}^{i_{k}} \alpha_{i} 1_{\left(u_{i}, a_{i}\right)}$. Then, we also have that $\tau_{k}^{\prime} \rightarrow \tau$ narrowly and $\operatorname{supp}\left(\tau_{k}^{\prime}\right) \rightarrow \operatorname{supp}(\tau)$ in the Hausdorff metric topology. In addition, for each $k \in \mathbb{N}$ and $(u, a) \in \operatorname{supp}\left(\hat{\tau}_{k}\right), a \in A_{u}$ since $(u, a) \in \operatorname{supp}(\tau)$ and $\tau$ is an equilibrium distribution for $\nu$; hence $\tau_{k, A}^{\prime}=\hat{\tau}_{k, A} \in E\left(\nu_{k}^{\prime}\right)$.

Note that $\tau_{k, A}^{\prime} \rightarrow \tau_{A}$. For each $k$ set

$$
\gamma_{k}=\max \left\{u\left(a^{\prime}, \tau_{k, A}^{\prime}\right)-u\left(a, \tau_{k, A}^{\prime}\right):(u, a) \in \operatorname{supp}\left(\tau_{k}^{\prime}\right), a^{\prime} \in A_{u}\right\}
$$

Then $\gamma_{k} \rightarrow 0$ because $\tau$ is an equilibrium distribution for $\nu, \tau_{k, A}^{\prime} \rightarrow \tau_{A}$ and $U$ is equicontinuous. 
Fix $k$ and write

$$
\tau_{k}^{\prime}=\sum_{i=1}^{i_{k}} \alpha_{i} 1_{\left(u_{i}, a_{i}\right)}=\sum_{i=1}^{i_{k}^{\prime}} \alpha_{i}^{\prime} \sum_{h=1}^{h_{i}} \alpha_{i, h} 1_{\left(u_{i}, a_{i, h}\right)}
$$

We have $u_{i}\left(a_{i, h}, \tau_{k, A}^{\prime}\right)>u_{i}\left(a, \tau_{k, A}^{\prime}\right)-\left(\gamma_{k}+\frac{1}{k}\right)$ for each $a \in A$ and each $(i, h)$. Put $\varepsilon_{k}=2\left(\gamma_{k}+\frac{1}{k}\right)$. Now there is $0<\delta_{k} \leq \varepsilon_{k} / 2$ such that for each $(i, h), u_{i}\left(a_{i, h}, \pi\right)>$ $u_{i}(a, \pi)-\varepsilon_{k}$ for each $\pi \in \bar{B}_{\delta_{k}}\left(\tau_{k, A}^{\prime}\right)$ and $a \in A$. For each $(i, h)$, choose a continuous function $\lambda_{i, h}: A \rightarrow \mathbb{R}_{+}$, with $\lambda_{i, h}\left(a_{i, h}\right)=2 \varepsilon_{k}, \lambda_{i, h} \leq 2 \varepsilon_{k}$ and $\lambda_{i, h}=0$ on $A_{i} \backslash B_{\delta_{k}}\left(a_{i, h}\right)$, and define $u_{i, h}$ by setting $u_{i, h}=u_{i}+\lambda_{i, h}$.

Thus, $u_{i, h}\left(a_{i, h}, \pi\right)>u_{i, h}(a, \pi)+\varepsilon_{k}$ for each $\pi \in \bar{B}_{\delta_{k}}\left(\tau_{k, A}^{\prime}\right)$ and $a \in A_{i} \backslash B_{\delta_{k}}\left(a_{i, h}\right)$. Choose $0<\eta_{k}<\delta_{k}$ such that $u_{i, h}\left(a^{\prime}, \pi\right)>u_{i, h}(a, \pi)+\varepsilon_{k}$ for each $\pi \in \bar{B}_{\delta_{k}}\left(\tau_{k, A}^{\prime}\right)$, $a^{\prime} \in A_{i} \cap \bar{B}_{\eta_{k}}\left(a_{i, h}\right)$ and $a \in A_{i} \backslash B_{\delta_{k}}\left(a_{i, h}\right)$.

Let $A_{i, h}^{k} \subseteq A_{i}$ be such that $\xi\left(A_{i, h}^{k}, A_{i}\right)<2 \varepsilon_{k}, a_{i, h} \in A_{i, h}$ and $d\left(a, a^{\prime}\right)>\varepsilon_{k}$ for each $a, a^{\prime} \in A_{i, h}^{k}$. To see that $A_{i, h}^{k}$ exists, let $\left\{a_{1}, \ldots, a_{m}\right\} \subseteq A_{i}$ be such that $a_{1}=a_{i, h}$ and $A_{i} \subseteq \cup_{j=1}^{m} B_{\varepsilon_{k}}\left(a_{j}\right)$; this is possible because $A_{i}$ is compact. Let $J^{1}=\{1, \ldots, m\}$ and, for each $1<j \leq m$, let $J^{j}=J^{j-1}$ if $d\left(a_{j}, a_{l}\right)>\varepsilon_{k}$ for all $l \in J^{j-1} \cap\{1, \ldots, j-1\}$ and $J^{j}=J^{j-1} \backslash\left\{a_{j}\right\}$ otherwise. Letting $A_{i, h}^{k}=\left\{a_{j}: j \in J^{m}\right\}$, we see that $d\left(a, a^{\prime}\right)>\varepsilon_{k}$ for all $a, a^{\prime} \in A_{i, h}^{k}$ and $A_{i} \subseteq \cup_{j \in J} B_{2 \varepsilon_{k}}\left(a_{j}\right)$.

Let $\bar{u}_{i, h}$ be the restriction of $u_{i, h}$ to $A_{i, h}^{k} \times \sum_{i=1}^{i_{k}^{\prime}} \alpha_{i}^{\prime} \sum_{h=1}^{h_{i}} \alpha_{i, h} M\left(A_{i, h}^{k}\right)$. Choose open neighborhoods $V_{i, h}$ of the functions $\bar{u}_{i, h}$ such that the family of these neighborhoods is disjoint and $A_{u} \cap \bar{B}_{\eta_{k}}\left(a_{i, h}\right) \neq \emptyset$ whenever $u \in V_{i, h}$; this latter requirement is possible because $u \mapsto A_{u}$ is lower hemicontinuous. Set $V_{k}=\bigcup_{i, h} V_{i, h}$ and define $s_{k}: V_{k} \rightarrow A$ by setting $s_{k}(u)=a_{i, h}$ if $u \in V_{i, h}$.

Define $\nu_{k}$ by setting

$$
\nu_{k}=\sum_{i=1}^{i_{k}^{\prime}} \alpha_{i}^{\prime} \sum_{h=1}^{h_{i}} \alpha_{i, h} 1_{\bar{u}_{i, h}} \text { and } \tau_{k}=\sum_{i=1}^{i_{k}^{\prime}} \alpha_{i}^{\prime} \sum_{h=1}^{h_{i}} \alpha_{i, h} 1_{\left(\bar{u}_{i, h}, a_{i, h}\right)} .
$$

Note that $\nu_{k} \circ s_{k}^{-1}=\tau_{k, A}=\tau_{k, A}^{\prime}$ and $E\left(\nu_{k}\right)=\sum_{i=1}^{i_{k}^{\prime}} \alpha_{i}^{\prime} \sum_{h=1}^{h_{i}} \alpha_{i, h} M\left(A_{i, h}^{k}\right)$. Thus, $\nu_{k} \in \mathcal{G}_{\varepsilon_{k}}^{*}$ and $\tau_{k} \in \mathcal{S}_{\varepsilon_{k}}\left(\nu_{k}\right)$.

Furthermore, $\xi\left(E\left(\nu_{k}\right), E\left(\nu_{k}^{\prime}\right)\right) \leq 2 \varepsilon_{k}$. Letting $\omega\left(2 \varepsilon_{k}\right)=\sup _{u \in U} \sup \{\mid u(a, \pi)-$ $\left.u\left(a^{\prime}, \pi^{\prime}\right) \mid: a, a^{\prime} \in A_{u}, \pi, \pi^{\prime} \in M(A), d\left(a, a^{\prime}\right) \leq 2 \varepsilon_{k}, \rho\left(\pi, \pi^{\prime}\right) \leq 2 \varepsilon_{k}\right\}$, we have that 
$d\left(\bar{u}_{i, h}, u_{i}\right) \leq 2 \varepsilon_{k}+\omega\left(2 \varepsilon_{k}\right)$ for each $(i, k)$ and that

$$
\rho\left(\tau_{k}, \tau_{k}^{\prime}\right) \leq \sum_{i=1}^{i_{k}^{\prime}} \alpha_{i}^{\prime} \sum_{h=1}^{h_{i}} \alpha_{i, h} \rho\left(1_{\left(\bar{u}_{i, h}, a_{i, h}\right)}, 1_{\left(u_{i}, a_{i, h}\right)}\right) \leq \sum_{i=1}^{i_{k}^{\prime}} \alpha_{i}^{\prime} \sum_{h=1}^{h_{i}} \alpha_{i, h} d\left(\bar{u}_{i, h}, u_{i}\right) .
$$

Thus $\rho\left(\tau_{k}, \tau_{k}^{\prime}\right) \rightarrow 0$ as $k \rightarrow \infty$, because $\varepsilon_{k} \rightarrow 0$. Because $\tau_{k}^{\prime} \rightarrow \tau$ narrowly, it follows that $\tau_{k} \rightarrow \tau$ narrowly. In particular, $\nu_{k} \rightarrow \nu$ narrowly. Together with the fact that $\operatorname{supp}\left(\nu_{k}^{\prime}\right) \rightarrow \operatorname{supp}(\nu)$ in the Hausdorff metric topology, the fact that $d\left(\bar{u}_{i, h}, u_{i}\right) \leq$ $\varepsilon_{k}+\omega\left(2 \varepsilon_{k}\right)$ for each $(i, h)$ and $\varepsilon_{k} \rightarrow 0$ also implies that $\operatorname{supp}\left(\nu_{k}\right) \rightarrow \operatorname{supp}(\nu)$ in that topology. Thus $\nu_{k} \rightarrow \nu$ in the topology of $\mathcal{G}$.

\section{A.4 Proofs}

\section{A.4.1 Proof of Lemma 1}

We establish the lemma for the general case where $A$ is compact and metric.

(Sufficiency) Let $U \subseteq \mathcal{U}$ be closed, bounded and equicontinuous, and $\left\langle u_{k}\right\rangle$ be a sequence in $U$. Let $B>0$ be such that $|u(a, \pi)| \leq B$ for each $u \in U, a \in A$ and $\pi \in M(A)$ such that $(a, \pi) \in \operatorname{dom}(u)$. As the space of the nonempty and compact subsets of $A \times M(A) \times[-B, B]$ is compact, we may assume that $\left\langle\operatorname{graph}\left(u_{k}\right)\right\rangle$ converges; let $C=\lim _{k} \operatorname{graph}\left(u_{k}\right)$. It thus remains to show that there exists $u \in \mathcal{U}$ such that $\operatorname{graph}(u)=C$ (indeed, we will then have that $u_{k} \rightarrow u$ and that $u \in U$ since $U$ is closed).

Note first that

$$
\operatorname{proj}_{A \times M(A)}(C)=\operatorname{proj}_{A}(C) \times \operatorname{proj}_{M(A)}(C) .
$$

We clearly have $\operatorname{proj}_{A \times M(A)}(C) \subseteq \operatorname{proj}_{A}(C) \times \operatorname{proj}_{M(A)}(C)$. Regarding the converse, let $(a, \pi) \in \operatorname{proj}_{A}(C) \times \operatorname{proj}_{M(A)}(C)$. Hence, there are $a^{\prime} \in A, \pi^{\prime} \in M(A)$ and $\alpha, \beta \in[-B, B]$ such that $\left(a, \pi^{\prime}, \alpha\right),\left(a^{\prime}, \pi, \beta\right) \in C$. Since $\operatorname{graph}\left(u_{k}\right) \rightarrow C$, there exist sequences $\left\langle\left(a_{k}, \pi_{k}^{\prime}, \alpha_{k}\right)\right\rangle$ and $\left\langle\left(a_{k}^{\prime}, \pi_{k}, \beta_{k}\right)\right\rangle$ such that $\left(a_{k}, \pi_{k}^{\prime}, \alpha_{k}\right) \rightarrow\left(a, \pi^{\prime}, \alpha\right)$, $\left(a_{k}^{\prime}, \pi_{k}, \beta_{k}\right) \rightarrow\left(a^{\prime}, \pi, \beta\right)$ and $\left(a_{k}, \pi_{k}^{\prime}, \alpha_{k}\right),\left(a_{k}^{\prime}, \pi_{k}, \beta_{k}\right) \in \operatorname{graph}\left(u_{k}\right)$ for all $k$ sufficiently large. Since, for each $k, \operatorname{dom}\left(u_{k}\right)=A_{k} \times M_{k}$ for some $A_{k} \subseteq A$ and $M_{k} \subseteq M(A)$, it follows that $\left(a_{k}, \pi_{k}, u_{k}\left(a_{k}, \pi_{k}\right)\right) \in \operatorname{graph}\left(u_{k}\right)$ for all $k$ sufficiently large. We may, 
taking a subsequence if necessary, assume that $\left\langle u_{k}\left(a_{k}, \pi_{k}\right)\right\rangle$ converges; letting $\gamma=$ $\lim _{k} u_{k}\left(a_{k}, \pi_{k}\right)$, it follows that $(a, \pi, \gamma) \in C$. Thus, $(a, \pi) \in \operatorname{proj}_{A \times M(A)}(C)$.

It remain to show that, for each $(a, \pi) \in \operatorname{proj}_{A}(C) \times \operatorname{proj}_{M(A)}(C)$, there is a unique $\alpha \in[-B, B]$ such that $(a, \pi, \alpha) \in C$; in fact, we can then set $\operatorname{dom}(u)=\operatorname{proj}_{A}(C) \times$ $\operatorname{proj}_{M(A)}(C)$ and $u(a, \pi)=\alpha$ for each $(a, \pi) \in \operatorname{proj}_{A}(C) \times \operatorname{proj}_{M(A)}(C)$. Suppose not; then there exist $(a, \pi) \in \operatorname{proj}_{A}(C) \times \operatorname{proj}_{M(A)}(C)$ and $\alpha, \beta \in[-B, B]$ such that $(a, \pi, \alpha),(a, \pi, \beta) \in C$. Then there exist sequences $\left\langle\left(a_{k}, \pi_{k}, \alpha_{k}\right)\right\rangle$ and $\left\langle\left(a_{k}^{\prime}, \pi_{k}^{\prime}, \beta_{k}\right)\right\rangle$ such that $\left(a_{k}, \pi_{k}, \alpha_{k}\right) \rightarrow(a, \pi, \alpha),\left(a_{k}^{\prime}, \pi_{k}^{\prime}, \beta_{k}\right) \rightarrow(a, \pi, \beta)$ and $\left(a_{k}, \pi_{k}, \alpha_{k}\right),\left(a_{k}^{\prime}, \pi_{k}^{\prime}, \beta_{k}\right) \in$ $\operatorname{graph}\left(u_{k}\right)$ for all $k$ sufficiently large. Pick $0<\varepsilon<|\alpha-\beta|$; the equicontinuity of $U$ then implies that, for all $k$ sufficiently large, $\left|\alpha_{k}-\beta_{k}\right|=\left|u_{k}\left(a_{k}, \pi_{k}\right)-u_{k}\left(a_{k}^{\prime}, \pi_{k}^{\prime}\right)\right|<\varepsilon$. Hence, we obtain that $|\alpha-\beta|=\lim _{k}\left|\alpha_{k}-\beta_{k}\right| \leq \varepsilon<|\alpha-\beta|$, a contradiction. This contradiction establishes our claim that, for each $(a, \pi) \in \operatorname{proj}_{A}(C) \times \operatorname{proj}_{M(A)}(C)$, there is a unique $\alpha \in[-B, B]$ such that $(a, \pi, \alpha) \in C$ and concludes the proof of the sufficiency part of the lemma.

(Necessity) Let $U \subseteq \mathcal{U}$ be compact. Suppose, in order to get a contradiction, that $U$ is not bounded. Then there exist sequences $\left\langle u_{k}\right\rangle,\left\langle a_{k}\right\rangle$ and $\left\langle\pi_{k}\right\rangle$, respectively in $U, A$ and $M(A)$, such that $\left|u_{k}\left(a_{k}, \pi_{k}\right)\right| \rightarrow \infty$. Since $U, A$ and $M(A)$ are compact, we may assume that there are $u \in U, a \in A$ and $\pi \in M(A)$ such that $u_{k} \rightarrow u$, $a_{k} \rightarrow a$ and $\pi_{k} \rightarrow \pi$. Therefore, $\lim _{k} u_{k}\left(a_{k}, \pi_{k}\right)=u(a, \pi)$ by Lemma 2 , contradicting $\left|u_{k}\left(a_{k}, \pi_{k}\right)\right| \rightarrow \infty$. This shows that $U$ is bounded.

Suppose next that $U$ is not equicontinuous. Then there exist $\varepsilon>0$ and sequences $\left\langle u_{k}\right\rangle,\left\langle a_{k}\right\rangle,\left\langle a_{k}^{\prime}\right\rangle,\left\langle\pi_{k}\right\rangle$ and $\left\langle\pi_{k}^{\prime}\right\rangle$, respectively in $U, A, A, M(A)$ and $M(A)$, such that the distance between $a_{k}$ and $a_{k}^{\prime}$ goes to zero, the distance between $\pi_{k}$ and $\pi_{k}^{\prime}$ goes to zero and $\left|u_{k}\left(a_{k}, \pi_{k}\right)-u_{k}\left(a_{k}^{\prime}, \pi_{k}^{\prime}\right)\right| \geq \varepsilon$. Since $U, A$ and $M(A)$ are compact, we may assume that there are $u \in U, a \in A$ and $\pi \in M(A)$ such that $u_{k} \rightarrow u, a_{k} \rightarrow a$ and $\pi_{k} \rightarrow \pi$. Therefore, $a_{k}^{\prime} \rightarrow a$ and $\pi_{k}^{\prime} \rightarrow \pi$ as well and, hence, $\lim _{k} u_{k}\left(a_{k}, \pi_{k}\right)=\lim _{k} u_{k}\left(a_{k}^{\prime}, \pi_{k}^{\prime}\right)=$ $u(a, \pi)$ by Lemma 2 . But then we obtain that $0=\lim _{k}\left|u_{k}\left(a_{k}, \pi_{k}\right)-u_{k}\left(a_{k}^{\prime}, \pi_{k}^{\prime}\right)\right| \geq \varepsilon$, a contradiction. This concludes the proof of the necessity part of the lemma. 


\section{A.4.2 Proof of Theorem 1}

(a) Recall that $\mathcal{G}^{*}$ is the subset of $\mathcal{G}$ consisting of those $\nu$ that have a strict equilibrium distribution, i.e. for some equilibrium distribution $\tau$ of $\nu, \#\left(\varphi\left(u, \tau_{A}\right)\right)=1$ for each $u \in \operatorname{supp}(\nu)$. As $A$ is finite, we can write $A=\left\{a_{1}, \ldots, a_{m}\right\}$.

(b) $\mathcal{G}^{*}$ is an open subset of $\mathcal{G}$. To see this, suppose $\nu \in \mathcal{G}^{*}$ and let $\tau$ be an equilibrium distribution for $\nu$ such that $\#\left(\varphi\left(u, \tau_{A}\right)\right)=1$ for each $u \in \operatorname{supp}(\nu)$. For each $j=1, \ldots, m$, let $H_{j}=\left\{u \in \operatorname{supp}(\nu): \varphi\left(u, \tau_{A}\right)=\left\{a_{j}\right\}\right\}$. We have that each $H_{j}$ is closed in $\operatorname{supp}(\nu)$, therefore compact $\operatorname{since} \operatorname{supp}(\nu)$ is. Indeed, if $\left\langle u_{k}\right\rangle$ is a sequence in $H_{j}$ converging to $u \in \mathcal{U}$, Lemma 2 implies that $u\left(a_{j}, \tau_{A}\right) \geq u\left(a, \tau_{A}\right)$ for each $a \in A$. But $\#\left(\varphi\left(u, \tau_{A}\right)\right)=1$ since $u \in \operatorname{supp}(\nu)$ and, hence, $\varphi\left(u, \tau_{A}\right)=\left\{a_{j}\right\}$.

We now claim that there is an open neighborhood $W$ of $\tau_{A}$ and, for each $j=$ $1, \ldots, m$, an open set $O_{j} \subseteq \mathcal{U}$, with $H_{j} \subseteq O_{j}$, such that $\varphi(u, \pi)=\left\{a_{j}\right\}$ for each $u \in O_{j}$ and each $\pi \in W$ such that $\left(a_{j}, \pi\right) \in \operatorname{dom}(u)$. If not, then there exists $j \in\{1, \ldots, m\}$, $\pi_{k} \rightarrow \tau_{A}, u_{k} \rightarrow u \in H_{j}$ and $a \in A$ such that $\left(a, \pi_{k}\right),\left(a_{j}, \pi_{k}\right) \in \operatorname{dom}\left(u_{k}\right)$ and $u_{k}\left(a, \pi_{k}\right) \geq$ $u_{k}\left(a_{j}, \pi_{k}\right)$ (recall that $A$ is finite). Lemma 2 implies that $u\left(a, \tau_{A}\right) \geq u\left(a_{j}, \tau_{A}\right)$, which is a contradiction since $u \in H_{j}$.

In particular, it follows by the above claim that $\operatorname{supp}(\nu) \subseteq \bigcup_{j=1}^{m} O_{j}$, and because the sets $O_{j}, j=1, \ldots, m$, are disjoint, we have a continuous function $h: \bigcup_{j=1}^{m} O_{j} \rightarrow A$ if we set $h(u)=a_{j}$ for each $u \in O_{j}$. Consequently, there is a neighborhood $V$ of $\nu$ in $\mathcal{G}$ such that $\nu^{\prime} \in V$ implies both that $\operatorname{supp}\left(\nu^{\prime}\right) \subseteq \bigcup_{j=1}^{m} O_{j}$ and that the distribution of $h$ on $A$ induced by $\nu^{\prime}$ belongs to $W$. Thus, for each $\nu^{\prime} \in V$, the distribution $\tau^{\prime}$ of $i d_{\mathcal{U}} \times h$ induced by $\nu^{\prime}$ is an equilibrium distribution with $\#\left(\varphi\left(u, \tau_{A}^{\prime}\right)\right)=1$ for each $u \in \operatorname{supp}\left(\nu^{\prime}\right)$. Thus $\mathcal{G}^{*}$ is open in $\mathcal{G}$.

(c) $\mathcal{G}^{*}$ is dense in $\mathcal{G}$. This follows by Lemma 7 .

(d) Let $\nu \in \mathcal{G}^{*}$ and let $\left\langle\left(I_{n}, G_{n}\right)\right\rangle_{n \in \mathbb{N}}$ be a sequence of games with $\nu_{\left(I_{n}, G_{n}\right)} \rightarrow \nu$ and $\#\left(I_{n}\right) \rightarrow \infty$. Let $\tau$ be an equilibrium distribution for $\nu$ such that $\#\left(\varphi\left(u, \tau_{A}\right)\right)=1$ for each $u \in \operatorname{supp}(\nu)$, and let $V, O_{j}, j=1, \ldots, m, W$ and $h$ be as in (b). As $\nu_{\left(I_{n}, G_{n}\right)} \rightarrow \nu$ there is an $N_{1} \in \mathbb{N}$ such that $\nu_{\left(I_{n}, G_{n}\right)} \in V$ for $n \geq N_{1}$, and in particular $\operatorname{supp}\left(\nu_{\left(I_{n}, G_{n}\right)}\right) \subseteq \bigcup_{j=1}^{m} O_{j}$. For such $n$, define $f_{n}: I_{n} \rightarrow A$ by setting $f_{n}(i)=h\left(G_{n}(i)\right)$ 
for each $i \in I_{n}$ and write $\tau_{f_{n}, A}$ for the distribution of $f_{n}$, i.e. for each $a \in A$,

$$
\tau_{f_{n}, A}(\{a\})=\frac{\#\left(\left\{i \in I_{n}: f_{n}(i)=a\right\}\right)}{\#\left(I_{n}\right)} .
$$

Then $\tau_{f_{n, A}}$ belongs to $W$, by the choice of $V$. In fact, $\tau_{f_{n, A}} \rightarrow \tau_{A}$ because $\nu_{\left(I_{n}, G_{n}\right)} \rightarrow \nu$ and $\tau_{f_{n, A}}$ is equal to the distribution of $h$ induced by $\nu_{\left(I_{n}, G_{n}\right)}$, i.e. $\tau_{f_{n}, A}=\nu_{\left(I_{n}, G_{n}\right)} \circ h^{-1}$.

For each $i \in I_{n}$ and $n \in \mathbb{N}$, let $e_{n, i}=\frac{1}{n-1} \sum_{j \in I_{n} \backslash\{i\}} 1_{f(j)}$ denote the action distribution induced by the choices of the players other than $i$. As $\#\left(I_{n}\right) \rightarrow \infty$, there is an $N \in \mathbb{N}$, with $N \geq N_{1}$, such that for $n \geq N$, $\left\|e_{n, i}-\tau_{A, f_{n}}\right\|$ is so small for each $i \in I_{n}$ that the fact that $\tau_{A, f_{n}} \rightarrow \tau_{A}$ implies that $e_{n, i} \in W$ for each $i \in I_{n}$. Thus, for such $n$ and each $i \in I_{n}, \varphi\left(G_{n}(i), e_{n, i}\right)=\left\{h\left(G_{n}(i)\right)\right\}=\left\{f_{n}(i)\right\}$, by the choice of $W$. Thus $f_{n}$ is a strict equilibrium for $G_{n}$ if $n \geq N$.

\section{A.4.3 Proof of Theorem 2}

(a) Let $\mu \in \mathcal{G}^{*}$ and let $\lambda$ be an equilibrium distribution for $\mu$ with $\#\left(\varphi\left(u, \lambda_{A}\right)\right)=1$ for each $u \in \operatorname{supp}(\mu)$. Let $\left\langle\left(I_{n}, G_{n}\right)\right\rangle_{n \in \mathbb{N}}$ be a sequence of finite-player games with $\nu_{\left(I_{n}, G_{n}\right)} \rightarrow \mu$ and $\#\left(I_{n}\right) \rightarrow \infty$. Such sequence exists by Lemma 6 .

Part (d) of the proof of Theorem 1 applies to yield an $N \in \mathbb{N}$ and a pure strategy Nash equilibrium $f_{n}$ of $\left(I_{n}, G_{n}\right)$ if $n \geq N$ such that, for some continuous function $h: O \rightarrow A$, where $O \subseteq \mathcal{U}$ is open with $\operatorname{supp}(\mu) \subseteq O$, we have both $f_{n}(i)=h\left(G_{n}(i)\right)$ for each $i \in I_{n}$ and $\lambda=\mu \circ\left(i d_{\mathcal{U}}, h\right)^{-1}$. Set $\lambda_{n}=\nu_{\left(I_{n}, G_{n}\right)} \circ\left(i d_{\mathcal{U}}, h\right)^{-1}$ for each $n \geq N$. Then, for $n \geq N, \lambda_{n}$ equals the distribution of $\left(G_{n}, f_{n}\right)$; because $\left(i d_{\mathcal{U}}, h\right)$ is continuous and $\nu_{\left(I_{n}, G_{n}\right)} \rightarrow \mu$, we have $\lambda_{n} \rightarrow \lambda$ narrowly.

(b) Putting (a) and Lemma 7 together proves the theorem.

\section{A.4.4 Proof of Theorem 4}

(a) Fix $\varepsilon>0$. Recall that $\mathcal{G}_{\varepsilon}^{*}$ is the subset of $\mathcal{G}$ consisting of those $\nu \in \mathcal{G}$ such that there is an open set $V \subseteq \mathcal{U}$, with $\operatorname{supp}(\nu) \subseteq V$, numbers $\eta$ and $\delta$, with $0<\eta<$ $\delta \leq \varepsilon / 2$, and a continuous function $s: V \rightarrow A$, with $A_{u} \cap \bar{B}_{\eta}(s(u)) \neq \emptyset$ for each $u \in V$, such that $u\left(a^{\prime}, \pi\right)>u(a, \pi)$ for all $u \in \operatorname{supp}(\nu), \pi \in \bar{B}_{\delta}\left(\nu \circ s^{-1}\right) \cap E(\nu)$, $a^{\prime} \in A_{u} \cap \bar{B}_{\eta}(s(u))$ and $a \in A_{u} \backslash B_{\delta}(s(u))$. 
(b) $\mathcal{G}_{\varepsilon}^{*}$ is open: Fix $\nu \in \mathcal{G}_{\varepsilon}^{*}$. Choose $V, \eta, \delta$ and $s$ witnessing that $\nu \in \mathcal{G}_{\varepsilon}^{*}$. Suppose $\nu_{n} \rightarrow \nu$ in $\mathcal{M}$. We need to show that $\nu_{n} \in \mathcal{G}_{\varepsilon}^{*}$ for all sufficiently large $n$. To see this, note first that $\operatorname{supp}\left(\nu_{n}\right) \subseteq V$ for large $n$. In particular, for such $n, A_{u} \cap \bar{B}_{\eta}(s(u)) \neq \emptyset$ for all $u \in \operatorname{supp}\left(\nu_{n}\right)$. Suppose by way of contradiction that along some subsequence $\left\langle\nu_{n_{k}}\right\rangle$ of $\left\langle\nu_{n}\right\rangle$ we can find $u_{n_{k}} \in \operatorname{supp}\left(\nu_{n_{k}}\right), \pi_{n_{k}} \in \bar{B}_{\delta}\left(\nu_{n_{k}} \circ s^{-1}\right) \cap E\left(\nu_{n_{k}}\right), a_{n_{k}}^{\prime} \in$ $A_{u_{n_{k}}} \cap \bar{B}_{\eta}\left(s\left(u_{n_{k}}\right)\right)$ and $a_{n_{k}} \in A_{u_{n_{k}}} \backslash B_{\delta}\left(s\left(u_{n_{k}}\right)\right)$ such that $u_{n_{k}}\left(a_{n_{k}}^{\prime}, \pi_{n_{k}}\right) \leq u_{n_{k}}\left(a_{n_{k}}, \pi_{n_{k}}\right)$. Then $\nu_{n_{k}} \circ s^{-1} \rightarrow \nu \circ s^{-1}$ because $s$ is continuous, and by compactness of $\operatorname{supp}(\nu), A$ and $M(A)$ we can assume, by Lemmas 4 and 5 , and passing to subsequences if necessary, that $u_{n_{k}} \rightarrow u \in \operatorname{supp}(\nu), \pi_{n_{k}} \rightarrow \pi \in E(\nu), a_{n_{k}}^{\prime} \rightarrow a^{\prime} \in A_{u}$ and $a_{n_{k}} \rightarrow a \in A_{u}$. Thus, $u\left(a^{\prime}, \pi\right) \leq u(a, \pi)$ by Lemma 2 . On the other hand, using continuity of $s$ and any metric, we see that $\pi \in \bar{B}_{\delta}\left(\nu \circ s^{-1}\right), a^{\prime} \in A_{u} \cap \bar{B}_{\eta}(s(u))$ and $a \in A_{u} \backslash B_{\delta}(s(u))$, thus getting a contradiction to the choice of $\eta, \delta$ and $s$. Thus $V, \eta, \delta$ and $s$ also witness that $\nu_{n} \in \mathcal{G}_{\varepsilon}^{*}$ for large $n$.

(c) $\mathcal{G}_{\varepsilon}^{*}$ is dense: This follows by Lemma 8 .

(d) Let $\nu \in \mathcal{G}_{\varepsilon}^{*}$ and choose $V, \eta, \delta$ and $s$ according to (a). Let $\left\langle\left(I_{n}, G_{n}\right)\right\rangle_{n \in \mathbb{N}}$ be a sequence of games with $\nu_{G_{n}} \rightarrow \nu$ and $\#\left(I_{n}\right) \rightarrow \infty$. Write $I_{n}=\left\{1, \ldots, k_{n}\right\}$ with $k_{n}=\#\left(I_{n}\right)$, and $u_{i}$ for $G_{n}(i)$. As $\nu_{\left(I_{n}, G_{n}\right)} \rightarrow \nu$, there is an $N_{1} \in \mathbb{N}$ such that $\operatorname{supp}\left(\nu_{\left(I_{n}, G_{n}\right)}\right) \in V$ for $n \geq N_{1}$. Thus $A_{u_{i}} \cap \bar{B}_{\eta}\left(s\left(u_{i}\right)\right) \neq \emptyset$ for each $i \in I_{n}$ if $n \geq N_{1}$. When each $i \in I_{n}$ is restricted to choose mixed strategies supported on $\bar{B}_{\delta}\left(s\left(u_{i}\right)\right)$, there is a mixed strategy Nash equilibrium $\sigma_{n}=\left(\sigma_{1}, \ldots, \sigma_{k_{n}}\right)$ for each $n \geq N_{1}$. Since $2 \delta \leq \varepsilon, \sigma_{n}$ is $\varepsilon$-concentrated. We need to show that there is an $N_{2} \geq N_{1}$ such that $\sigma_{n}$ is a mixed strategy Nash equilibrium (when players are unrestricted) if $n \geq N_{2}$.

To this end, note first that for each $n \geq N_{1}$ and $i \in I_{n}$, the expected payoff in the equilibrium $\sigma_{n}$ is at least as large as the expected payoff at any $a \in A_{u_{i}} \cap \bar{B}_{\delta}\left(s\left(u_{i}\right)\right)$ against $\sigma_{n,-i}$. It therefore suffices to show that, for all $n$ larger than some $N_{2} \geq N_{1}$ and each $i \in I_{n}$, the expected payoff at some $a_{i}^{*} \in A_{u_{i}} \cap \bar{B}_{\eta}\left(s\left(u_{i}\right)\right)$ against $\sigma_{n,-i}$ is at least as large as the expected payoff against $\sigma_{n,-i}$ at any $a \in A_{u_{i}} \backslash \bar{B}_{\delta}\left(s\left(u_{i}\right)\right)$. Because $\operatorname{supp}\left(\sigma_{n, j}\right) \subseteq \bar{B}_{\delta}\left(s\left(u_{j}\right)\right)$ for all $j \in I_{n}, n \geq N_{1}$, this follows if we can show that there is an $N_{2} \geq N_{1}$ such that if $n \geq N_{2}$, then for any $i \in I_{n}, u_{i}\left(f(i), \frac{1}{k_{n}-1} \sum_{j \in I_{n} \backslash\{i\}} 1_{f(j)}\right) \geq$ $u_{i}\left(a, \frac{1}{k_{n}-1} \sum_{j \in I_{n} \backslash\{i\}} 1_{f(j)}\right)$ for any map $f: I_{n} \rightarrow A$, with $f(j) \in \bar{B}_{\delta}\left(s\left(u_{j}\right)\right) \cap A_{u_{j}}$ for 
each $j \in I_{n}, f(i) \in \bar{B}_{\eta}\left(s\left(u_{i}\right)\right) \cap A_{u_{i}}$ and any $a \in A_{u_{i}} \backslash \bar{B}_{\delta}\left(s\left(u_{i}\right)\right)$.

If no such $N_{2}$ exists, then there is a subsequence $\left\langle\left(I_{h}, G_{h}\right)\right\rangle$ of $\left\langle\left(I_{n}, G_{n}\right)\right\rangle, i_{h} \in I_{h}$, a map $f_{h}: I_{h} \rightarrow A$, with $f_{h}(i) \in \bar{B}_{\delta}\left(s\left(u_{i}\right)\right) \cap A_{u_{i}}$ for each $i \in I_{h}, f_{h}\left(i_{h}\right) \in \bar{B}_{\eta}\left(s\left(u_{i_{h}}\right)\right) \cap$ $A_{u_{i_{h}}}$ and $a_{h} \in A_{u_{i_{h}}} \backslash \bar{B}_{\delta}\left(s\left(u_{i_{h}}\right)\right)$ such that

$$
u_{i_{h}}\left(f_{h}\left(i_{h}\right), \frac{1}{k_{h}-1} \sum_{i \in I_{h} \backslash\left\{i_{h}\right\}} 1_{f_{h}(i)}\right)<u_{i_{h}}\left(a_{h}, \frac{1}{k_{h}-1} \sum_{i \in I_{h} \backslash\left\{i_{h}\right\}} 1_{f_{h}(i)}\right)
$$

for each $h$. Write $\nu_{h, i}$ for the distribution of the payoff functions of the players in $I_{h} \backslash\left\{i_{h}\right\}$ and note that $\nu_{h, i} \rightarrow \nu$. Since $f_{h}(i) \in \bar{B}_{\delta}\left(s\left(u_{i}\right)\right)$ for each $i \in I_{h}$, we have

$$
\frac{1}{k_{h}-1} \sum_{i \in I_{h} \backslash\left\{i_{h}\right\}} 1_{f(i)} \in \bar{B}_{\delta}\left(\frac{1}{k_{h}-1} \sum_{i \in I_{h} \backslash\left\{i_{h}\right\}} 1_{s\left(u_{i}\right)}\right)=\bar{B}_{\delta}\left(\nu_{h, i} \circ s^{-1}\right)
$$

for each $h$. Passing to subsequences, if necessary, we can conclude by Lemmas 2, 4 and 5 that, in the limit as $h \rightarrow \infty$, there are $u \in \operatorname{supp}(\nu), \pi \in \bar{B}_{\delta}\left(\nu \circ s^{-1}\right) \cap E(\nu)$, $a^{\prime} \in A_{u} \cap \bar{B}_{\eta}(s(u))$ and $a \in A_{u} \backslash B_{\delta}(s(u))$ such that $u\left(a^{\prime}, \pi\right) \leq u(a, \pi)$, contradicting the choices of $s, \eta$ and $\delta$.

\section{A.4.5 Proof of Theorem 5}

(a) Let $\varepsilon>0, \mu \in \mathcal{G}_{\varepsilon}^{*}$ and $\lambda \in \mathcal{S}_{\varepsilon}(\mu)$ (see the text before Lemma 8 for the definition of $\left.\mathcal{S}_{\varepsilon}(\mu)\right)$ be such that $d\left(a, a^{\prime}\right)>\varepsilon$ for all $a, a^{\prime} \in A_{u}$ and $u \in \operatorname{supp}(\mu)$. Let $\left\langle\left(I_{n}, G_{n}\right)\right\rangle_{n \in \mathbb{N}}$ be a sequence of finite-player games with $\nu_{\left(I_{n}, G_{n}\right)} \rightarrow \mu, \#\left(I_{n}\right) \rightarrow \infty$ and $\operatorname{supp}\left(\nu_{\left(I_{n}, G_{n}\right)}\right) \subseteq$ $\operatorname{supp}(\mu)$. Such sequence exists by Lemma 6 .

Let $V, \eta, \delta$ and $s$ witness that $\lambda \in \mathcal{S}_{\varepsilon}(\mu)$ and note that $\operatorname{supp}\left(\nu_{\left(I_{n}, G_{n}\right)}\right) \subseteq V$ for all $n$ sufficiently large. Part (d) of the proof of Theorem 4 applies to yield an $N^{\prime} \in \mathbb{N}$ such that, for each $n \geq N^{\prime},\left(I_{n}, G_{n}\right)$ has a mixed strategy Nash equilibrium $\sigma_{n}$ such that $\sigma_{n}$ is $\varepsilon$-concentrated and $\operatorname{supp}\left(\sigma_{n, i}\right) \subseteq \bar{B}_{\delta}\left(s\left(u_{i}\right)\right)$ for each $i \in I_{n}$. Since $d\left(a, a^{\prime}\right)>\varepsilon$ for all $a, a^{\prime} \in A_{u}$ and $u \in \operatorname{supp}\left(\nu_{\left(I_{n}, G_{n}\right)}\right)$, it follows that $\sigma_{n}$ is pure.

We have that $\nu_{\left(I_{n}, G_{n}\right)} \circ\left(i d_{\mathcal{U}}, s\right)^{-1} \rightarrow \lambda$ since $\lambda=\mu \circ\left(i d_{\mathcal{U}}, s\right)^{-1}$ and $\left(i d_{\mathcal{U}}, s\right)$ : $V \rightarrow V \times A$ is continuous. Thus, there is $N \geq N^{\prime}$ such that, for each $n \geq N$, $\rho\left(\nu_{\left(I_{n}, G_{n}\right)} \circ\left(i d_{\mathcal{U}}, s\right)^{-1}, \lambda\right)<\frac{1}{n}$. Moreover, it follows that

$$
\rho\left(\sum_{i \in I_{n}} 1_{\left(G_{n}(i), \sigma_{n}(i)\right)} / \#\left(I_{n}\right), \sum_{i \in I_{n}} 1_{\left(G_{n}(i), s(i)\right)} / \#\left(I_{n}\right)\right) \leq \delta .
$$


Since $\nu_{\left(I_{n}, G_{n}\right)} \circ\left(i d_{\mathcal{U}}, s\right)^{-1}=\sum_{i \in I_{n}} 1_{\left(G_{n}(i), s(i)\right)} / \#\left(I_{n}\right)$, it follows that, for each $n \geq N$,

$$
\rho\left(\sum_{i \in I_{n}} 1_{\left(G_{n}(i), \sigma_{n}(i)\right)} / \#\left(I_{n}\right), \lambda\right)<\delta+\frac{1}{n} .
$$

(b) Putting (a) and Lemma 8 together proves the theorem.

\section{A.5 First-price auctions}

We shall use the following notation: For each $v \in \mathbb{R}_{+}$, let $u_{v}: A \times M(A) \rightarrow \mathbb{R}$ be defined by $u_{v}(a, \pi)=\Phi(a, \pi)(v-a)$ for each $(a, \pi) \in A \times M(A)$. Moreover, we write $\varphi(v, \pi)$ instead of $\varphi\left(u_{v}, \pi\right)$. Also, for each $\pi \in M(A)$, we let $A_{0}(\pi)=\{a \in A$ : $\Phi(a, \pi)=0\}$.

Lemma 9. If $\nu \in \tilde{\mathcal{G}}$ and $\tau$ is an equilibrium distribution of $\nu$, then $\tau_{A}\left(p^{*}\left(\tau_{A}\right)\right)>0$ and $\Phi\left(p^{*}\left(\tau_{A}\right), \tau_{A}\right)>0$.

Proof. Indeed, if $\tau_{A}\left(p^{*}\left(\tau_{A}\right)\right)=0$, then $\sum_{p \geq p^{*}\left(\tau_{A}\right)+1} \tau_{A}(p)=\sum_{p \geq p^{*}\left(\tau_{A}\right)} \tau_{A}(p) \geq 1 / k$ and, hence, $\max \left\{p \in A: \sum_{p^{\prime} \geq p} \tau_{A}\left(p^{\prime}\right)\right\}>p^{*}\left(\tau_{A}\right)$, a contradiction. Moreover, if $\Phi\left(p^{*}\left(\tau_{A}\right), \tau_{A}\right)=0$, then $\sum_{p \geq p^{*}\left(\tau_{A}\right)+1} \tau_{A}(p)=1 / k$ and we reach the same contradiction.

Lemma 10. $\Phi$ is continuous at each $(a, \pi) \in A \times M(A)$ such that $\sum_{p \geq p^{*}(\pi)} \pi(p)>$ $1 / k$.

Proof. This is clear, because if $\left\langle\pi_{j}\right\rangle$ is a sequence in $M(A)$ converging to such $\pi$, we have that $p^{*}\left(\pi_{j}\right) \rightarrow p^{*}(\pi)$.

Given $\nu \in \tilde{\mathcal{G}}$ and an equilibrium distribution $\tau$ of $\nu$, we say that $\tau$ is essentially strict if $\sum_{p \geq p^{*}\left(\tau_{A}\right)} \tau_{A}(p)>1 / k$ and, for each $u \in \operatorname{supp}(\nu)$,

$$
\varphi\left(u, \tau_{A}\right)= \begin{cases}\{a\} & \text { if } \Phi\left(a, \tau_{A}\right)>0 \text { for some } a \in A \text { with }(u, a) \in \operatorname{supp}(\tau), \\ A_{0}\left(\tau_{A}\right) & \text { otherwise. }\end{cases}
$$

Let $\tilde{\mathcal{G}}^{*}$ be the set of $\nu \in \tilde{\mathcal{G}}$ that have an essentially strict equilibrium distribution. 
Lemma 11. If $\nu \in \tilde{\mathcal{G}}$ and $\tau$ is an equilibrium distribution of $\nu$, then there exists $\left\langle\left(\nu_{n}, \tau_{n}\right)\right\rangle_{n \in \mathbb{N}}$ such that $\nu_{n} \in \tilde{\mathcal{G}}^{*}$ and $\tau_{n}$ is an essentially strict equilibrium distribution of $\nu_{n}$ for all $n \in \mathbb{N}, \nu_{n} \rightarrow \nu$ in the topology of $\tilde{\mathcal{G}}$ and $\tau_{n} \rightarrow \tau$ narrowly.

Proof. Let $\nu \in \tilde{\mathcal{G}}$ and $\tau$ be an equilibrium distribution of $\nu$. Given $\nu^{\prime} \in \tilde{\mathcal{G}}$ and an equilibrium distribution $\tau^{\prime}$ of $\nu^{\prime}$, we say that $\tau$ is almost strict if, for each $u \in \operatorname{supp}(\nu)$,

$$
\varphi\left(u, \tau_{A}\right)= \begin{cases}\{a\} & \text { if } \Phi\left(a, \tau_{A}\right)>0 \text { for some } a \in A \text { with }(u, a) \in \operatorname{supp}(\tau) \\ A_{0}\left(\tau_{A}\right) & \text { otherwise. }\end{cases}
$$

Therefore, $\tau$ is essentially strict if and only if $\tau$ is almost strict and such that $\sum_{p \geq p^{*}\left(\tau_{A}\right)} \tau_{A}(p)>1 / k$. To simplify the notation, we will write $p^{*}$ for $p^{*}\left(\tau_{A}\right)$ and, for each $a \in A, \Phi(a)$ for $\Phi\left(a, \tau_{A}\right)$.

(a) We first show that there exists $\left\langle\left(\nu_{n}, \tau_{n}\right)\right\rangle_{n \in \mathbb{N}}$ such that $\tau_{n}$ is an almost strict equilibrium distribution of $\nu_{n}$ for all $n \in \mathbb{N}, \nu_{n} \rightarrow \nu$ in the topology of $\mathcal{G}$ and $\tau_{n} \rightarrow \tau$ narrowly.

To see this, first note that $\operatorname{supp}\left(\tau_{A}\right) \subseteq\left\{p^{*}, p^{*}+1\right\} \cup A_{0}\left(\tau_{A}\right)$ since it does not pay to bid more that $p^{*}+1$ as $\Phi\left(p^{*}+1\right)=1$.

Consider first the case where $p^{*}=0$. In this case $A_{0}\left(\tau_{A}\right)=\emptyset$ by Lemma 9 and there can only be indifference between $p^{*}$ and $p^{*}+1$, which happens for $\bar{v} \in \mathbb{R}_{+}$ satisfying $\bar{v}-1=\Phi(0) \bar{v}$, i.e. $\bar{v}=\frac{1}{1-\Phi(0)}$ (of course, if $\Phi(0)=1$, then $1=p^{*}+1$ is not in the support of $\tau_{A}$ because choosing 0 is strictly better than choosing 1 for any $v \in \mathbb{R}_{+}$; in this case, $\tau$ is essentially strict). Thus, for $\varepsilon>0$, define

$$
\tau_{\varepsilon}=\tau+\tau(\{(\bar{v}, 0)\})\left(1_{(\bar{v}-\varepsilon, 0)}-1_{(\bar{v}, 0)}\right)+\tau(\{(\bar{v}, 1)\})\left(1_{(\bar{v}+\varepsilon, 1)}-1_{(\bar{v}, 1)}\right) .
$$

By letting $\nu_{\varepsilon}=\tau_{\varepsilon, \mathbb{R}_{+}}$and $\varepsilon \rightarrow 0$, we obtain the desired conclusion.

Consider next the case where $p^{*}>0$. Indifference can occur between any element of $A_{0}\left(\tau_{A}\right)$ and $p^{*}$ when $v \in \mathbb{R}_{+}$is such that $0=\Phi\left(p^{*}\right)\left(v-p^{*}\right)$, i.e. $v=p^{*}$. Moreover, indifference can occur between $p^{*}$ and $p^{*}+1$ when $v \in \mathbb{R}_{+}$is such that $v-p^{*}-1=$ $\Phi\left(p^{*}\right)\left(v-p^{*}\right)$, i.e. $v=p^{*}+\frac{1}{1-\Phi\left(p^{*}\right)}$ (as before, we can discard this last indifference if 
$\left.\Phi\left(p^{*}\right)=1\right)$. Thus, for $\varepsilon>0$, define

$$
\begin{aligned}
\tau_{\varepsilon}=\tau & +\sum_{a \in A_{0}\left(\tau_{A}\right)} \tau\left(\left\{\left(p^{*}, a\right)\right\}\right)\left(1_{\left(p^{*}-\varepsilon, a\right)}-1_{\left(p^{*}, a\right)}\right) \\
& +\tau\left(\left\{\left(p^{*}, p^{*}\right)\right\}\right)\left(1_{\left(p^{*}+\varepsilon, p^{*}\right)}-1_{\left(p^{*}, p^{*}\right)}\right) \\
& +\tau\left(\left\{\left(p^{*}+\frac{1}{1-\Phi\left(p^{*}\right)}, p^{*}\right)\right\}\right)\left(1_{\left(p^{*}+\frac{1}{1-\Phi\left(p^{*}\right)}-\varepsilon, p^{*}\right)}-1_{\left(p^{*}+\frac{1}{1-\Phi\left(p^{*}\right)}, p^{*}\right)}\right) \\
& +\tau\left(\left\{\left(p^{*}+\frac{1}{1-\Phi\left(p^{*}\right)}, p^{*}+1\right)\right\}\right)\left(1_{\left(p^{*}+\frac{1}{1-\Phi\left(p^{*}\right)}+\varepsilon, p^{*}+1\right)}-1_{\left(p^{*}+\frac{1}{1-\Phi\left(p^{*}\right)}, p^{*}+1\right)}\right) .
\end{aligned}
$$

By letting $\nu_{\varepsilon}=\tau_{\varepsilon, \mathbb{R}_{+}}$and $\varepsilon \rightarrow 0$, we obtain the desired conclusion.

(b) By part (a), we may assume that $\tau$ is almost strict and that $\sum_{p \geq p^{*}} \tau_{A}(p)=$ $1 / k$. Because $\operatorname{supp}\left(\tau_{A}\right) \subseteq A_{0}\left(\tau_{A}\right) \cup\left\{p^{*}, p^{*}+1\right\}$, we have that $\tau_{A}\left(p^{*}\right)+\tau_{A}\left(p^{*}+1\right)=1 / k$ and, hence, $\Phi\left(p^{*}\right)=1$. Since $\tau$ is almost strict, it then follows that $\tau_{A}\left(p^{*}+1\right)=0$, $\frac{1}{k}=\tau_{A}\left(p^{*}\right)=\nu\left(\left(p^{*}, \infty\right)\right)$ and $1-\frac{1}{k}=\nu\left(\left[0, p^{*}\right)\right)$. Pick $v^{*} \in \operatorname{supp}(\nu) \cap\left(p^{*}, \infty\right)$ and define, for a given $\varepsilon>0$,

$$
\tau_{\varepsilon}=\varepsilon 1_{\left(v^{*}, p^{*}\right)}+(1-\varepsilon) \tau
$$

Then $\tau_{\varepsilon, A}\left(p^{*}\right)=\varepsilon+(1-\varepsilon) \frac{1}{k}>\frac{1}{k}$ and $p^{*}\left(\tau_{\varepsilon, A}\right)=p^{*}$. In addition, $\Phi\left(p^{*}, \tau_{\varepsilon, A}\right)=$ $\frac{1}{1+(k-1) \varepsilon}$ and, therefore, to check that $\tau_{\varepsilon}$ is essentially strict, it suffices to show that $u_{v}\left(p^{*}, \tau_{\varepsilon, A}\right)>u_{v}\left(0, \tau_{\varepsilon, A}\right)=0$ and $u_{v}\left(p^{*}, \tau_{\varepsilon, A}\right)>u_{v}\left(p^{*}+1, \tau_{\varepsilon, A}\right)$ for each $v \in \operatorname{supp}(\nu) \cap$ $\left(p^{*}, \infty\right)$. The former holds since $u_{v}\left(p^{*}, \tau_{\varepsilon, A}\right)=\Phi\left(p^{*}, \tau_{\varepsilon, A}\right)\left(v-p^{*}\right)>0$ since $v \in\left(p^{*}, \infty\right)$. The latter holds for all sufficiently small $\varepsilon>0$ : Indeed, letting $\bar{v}=\max \operatorname{supp}(\nu)$, pick $\varepsilon>0$ such that $\Phi\left(p^{*}, \tau_{\varepsilon, A}\right)>1-\frac{1}{\bar{v}-p^{*}}$. Hence, for each $v \in \operatorname{supp}(\nu) \cap\left(p^{*}, \infty\right)$, $\Phi\left(p^{*}, \tau_{\varepsilon, A}\right)>1-\frac{1}{v-p^{*}}$ and, thus, $u_{v}\left(p^{*}, \tau_{\varepsilon, A}\right)=\Phi\left(p^{*}, \tau_{\varepsilon, A}\right)\left(v-p^{*}\right)>v-p^{*}-1=$ $u_{v}\left(p^{*}+1, \tau_{\varepsilon, A}\right)$. By letting $\nu_{\varepsilon}=\tau_{\varepsilon, \mathbb{R}_{+}}$and $\varepsilon \rightarrow 0$, we obtain the desired conclusion.

Lemma 12. If $\nu \in \tilde{\mathcal{G}}$ and $\tau$ is an equilibrium distribution of $\nu$, then there exists $\left\langle\left(\nu_{n}, \tau_{n}\right)\right\rangle_{n \in \mathbb{N}}$ such that $\nu_{n} \rightarrow \nu$ in the topology of $\tilde{\mathcal{G}}, \tau_{n} \rightarrow \tau$ narrowly and, for each $n \in \mathbb{N}, \nu_{n} \in \tilde{\mathcal{G}}^{*}, \tau_{n}$ is an essentially strict equilibrium distribution of $\nu_{n}, \operatorname{supp}\left(\tau_{n}\right)$ is finite and

$$
\tau_{n}=\sum_{v \in \operatorname{supp}\left(\nu_{n}\right)} \nu_{n}(v) 1_{\left(v, a_{v}\right)},
$$

where $a_{v} \in A$ for each $v \in \operatorname{supp}\left(\nu_{n}\right)$. 
Proof. Let $\nu \in \tilde{\mathcal{G}}$ and $\tau$ be an equilibrium distribution of $\nu$. Due to Lemma 11, we may assume that $\tau$ is essentially strict. As before, we will write $p^{*}$ for $p^{*}\left(\tau_{A}\right)$ and, for each $a \in A, \Phi(a)$ for $\Phi\left(a, \tau_{A}\right)$. Thus,

$$
\tau=\sum_{a \in A_{0}\left(\tau_{A}\right)} \tau_{A}(a) \tau_{a}+\tau_{A}\left(p^{*}\right) \tau_{p^{*}}+\tau_{A}\left(p^{*}+1\right) \tau_{p^{*}+1}
$$

where, for each $a \in A_{0}\left(\tau_{A}\right) \cup\left\{p^{*}, p^{*}+1\right\}, \tau_{a}$ is the Borel probability measure on $\mathbb{R}_{+} \times A$ defined by setting, for each Borel $B \subseteq \mathbb{R}_{+}$and $a^{\prime} \in A$,

$$
\tau_{a}\left(B \times\left\{a^{\prime}\right\}\right)=\frac{\tau(B \times\{a\})}{\tau_{A}(a)}
$$

if $a^{\prime}=a$ and $\tau_{a}\left(B \times\left\{a^{\prime}\right\}\right)=0$ if $a^{\prime} \neq a$. Since $\tau$ is essentially strict, we have that $\operatorname{supp}\left(\tau_{a}\right) \subseteq\left[0, p^{*}\right] \times A_{0}\left(\tau_{A}\right), \operatorname{supp}\left(\tau_{p^{*}}\right) \subseteq\left[p^{*}, p^{*}+\left(1-\Phi\left(p^{*}\right)\right)^{-1}\right] \times\left\{p^{*}\right\}$ and $\operatorname{supp}\left(\tau_{p^{*}+1}\right) \subseteq\left[p^{*}+\left(1-\Phi\left(p^{*}\right)\right)^{-1}, \infty\right) \times\left\{p^{*}+1\right\}$. For each $a \in A_{0}\left(\tau_{A}\right) \cup\left\{p^{*}, p^{*}+1\right\}$, there exists $\left\langle\tau_{n, a}\right\rangle$ such that (i) $\tau_{n, a} \rightarrow \tau_{a}$ narrowly, (ii) the sequence of the marginals of $\tau_{n, a}$ on $\mathbb{R}_{+}$converges to the marginal of $\tau_{a}$ on $\mathbb{R}_{+}$in the topology of $\tilde{\mathcal{G}}$, (iii) $\operatorname{supp}\left(\tau_{n, a}\right) \cap$ $\left\{p^{*}, p^{*}+\left(1-\Phi\left(p^{*}\right)\right)^{-1}\right\}=\emptyset$ and (iv) $\operatorname{supp}\left(\tau_{n, a}\right) \cap \operatorname{supp}\left(\tau_{n, a^{\prime}}\right)=\emptyset$ for each $a^{\prime} \neq a$. Thus, put

$$
\tau_{n}=\sum_{a \in A_{0}\left(\tau_{A}\right)} \tau_{A}(a) \tau_{n, a}+\tau_{A}\left(p^{*}\right) \tau_{n, p^{*}}+\tau_{A}\left(p^{*}+1\right) \tau_{n, p^{*}+1}
$$

for each $n \in \mathbb{N}$.

\section{A.5.1 Proof of Theorem 6}

(a) Recall that $\tilde{\mathcal{G}}^{*}$ is the set of those $\nu \in \tilde{\mathcal{G}}$ that have an essentially strict equilibrium distribution $\tau$; thus, $\sum_{p \geq p^{*}\left(\tau_{A}\right)} \tau_{A}(p)>1 / k$ and, for each $u \in \operatorname{supp}(\nu)$,

$$
\varphi\left(u, \tau_{A}\right)= \begin{cases}\{a\} & \text { if } \Phi\left(a, \tau_{A}\right)>0 \text { for some } a \in A \text { with }(u, a) \in \operatorname{supp}(\tau) \\ A_{0}\left(\tau_{A}\right) & \text { otherwise. }\end{cases}
$$

As $A$ is finite, we can write $A=\left\{a_{1}, \ldots, a_{m}\right\}$.

(b) $\tilde{\mathcal{G}}^{*}$ is an open subset of $\tilde{\mathcal{G}}$. To see this, suppose $\nu \in \tilde{\mathcal{G}}^{*}$ and let $\tau$ be an essentially strict equilibrium distribution for $\nu$. In particular, $\sum_{p \geq p^{*}\left(\tau_{A}\right)} \tau_{A}(p)>1 / k$ and, hence, $\Phi$ is continuous at $\left(a, \tau_{A}\right)$ for each $a \in A$ by Lemma 10 . 
We may assume that $\tau_{A}\left(A_{0}\left(\tau_{A}\right)\right)=\tau_{A}(0)$. Indeed, let $f: A \rightarrow A$ be defined by setting, for each $a \in A$,

$$
f(a)= \begin{cases}0 & \text { if } \Phi\left(a, \tau_{A}\right)=0, \\ a & \text { otherwise. }\end{cases}
$$

We then have that $\hat{\tau}:=\tau \circ\left(i d_{\mathbb{R}_{+}} \times f\right)^{-1}$ is also essentially strict since, by Lemma 9 , $p^{*}\left(\tau_{A}\right) \notin A_{0}\left(\tau_{A}\right)$ and, hence, $p^{*}\left(\hat{\tau}_{A}\right)=p^{*}\left(\tau_{A}\right)$. And, clearly, $\hat{\tau}_{A}\left(A_{0}\left(\tau_{A}\right)\right)=\hat{\tau}_{A}(0)$.

For each $j=1, \ldots, m$, let $H_{j}=\left\{v \in \operatorname{supp}(\nu): \varphi\left(v, \tau_{A}\right)=\left\{a_{j}\right\}\right\}$; let $H_{m+1}=$ $\operatorname{supp}(\nu) \backslash \bigcup_{j=1}^{m} H_{j}$. We have that each $H_{j}$ is closed in $\operatorname{supp}(\nu)$, therefore compact since $\operatorname{supp}(\nu)$ is. Indeed, using the continuity of $\Phi$ at $\left(a, \tau_{A}\right)$ for each $a \in A$, if $\left\langle v_{k}\right\rangle$ is a sequence in $H_{j}$ converging to $v \in \mathbb{R}_{+}$, then $u_{v}\left(a_{j}, \tau_{A}\right) \geq u_{v}\left(a, \tau_{A}\right)$ for each $a \in A$, letting $a_{m+1}$ be any element of $A_{0}\left(\tau_{A}\right)$. Since $v \in \operatorname{supp}(\nu)$, the properties of $\tau$ imply that $\varphi\left(v, \tau_{A}\right)=\left\{a_{j}\right\}$ if $j \neq m+1$ and $\varphi\left(v, \tau_{A}\right)=A_{0}\left(\tau_{A}\right)$ if $j=m+1$.

Using again the continuity of $\Phi$ at $\left(a, \tau_{A}\right)$ for each $a \in A$, it follows that there is an open neighborhood $W$ of $\tau_{A}$ and, for each $j=1, \ldots, m+1$, an open set $O_{j} \subseteq \mathbb{R}_{+}$, with $H_{j} \subseteq O_{j}$, such that $p^{*}(\pi)=p^{*}\left(\tau_{A}\right), \sum_{p \geq p^{*}\left(\tau_{A}\right)} \pi(p)>1 / k$ and

$$
\varphi(v, \pi)= \begin{cases}\left\{a_{j}\right\} & \text { if } j \neq m+1, \\ A_{0}\left(\tau_{A}\right) & \text { if } j=m+1\end{cases}
$$

for each $v \in O_{j}$ and each $\pi \in W$.

In particular, it follows by the above claim that $\operatorname{supp}(\nu) \subseteq \bigcup_{j=1}^{m+1} O_{j}$, and because the sets $O_{j}, j=1, \ldots, m+1$, are disjoint, we have a continuous function $h: \bigcup_{j=1}^{m+1} O_{j} \rightarrow A$ if we set $h(u)=a_{j}$ for each $u \in O_{j}$ and $j \neq m+1$ and $h(u)=0$ for each $u \in O_{m+1}$. Consequently, there is a neighborhood $V$ of $\nu$ in $\tilde{\mathcal{G}}$ such that $\nu^{\prime} \in V$ implies both that $\operatorname{supp}\left(\nu^{\prime}\right) \subseteq \bigcup_{j=1}^{m+1} O_{j}$ and that the distribution of $h$ on $A$ induced by $\nu^{\prime}$ belongs to $W$. Thus, for each $\nu^{\prime} \in V$, the distribution $\tau^{\prime}$ of $i d_{\mathcal{U}} \times h$ induced by $\nu^{\prime}$ is an equilibrium distribution witnessing that $\nu^{\prime} \in \tilde{\mathcal{G}}^{*}$. Thus $\tilde{\mathcal{G}}^{*}$ is open in $\tilde{\mathcal{G}}$.

(c) $\tilde{\mathcal{G}}^{*}$ is dense in $\tilde{\mathcal{G}}$. This follows by Lemma 11.

(d) The remainder of the proof is as part (d) of the proof of Theorem 1. 


\section{A.5.2 Proof of Theorem 7}

The proof is analogous to the proof of Theorem 2 with the following modification. Let $\nu \in \tilde{\mathcal{G}}^{*}$ and let $\tau$ be an essentially strict equilibrium distribution for $\nu$ such that $\operatorname{supp}(\tau)$ is finite and

$$
\tau=\sum_{v \in \operatorname{supp}(\nu)} \nu(v) 1_{\left(v, a_{v}\right)},
$$

where $a_{v} \in A$ for each $v \in \operatorname{supp}(\nu)$. We will write $p^{*}$ for $p^{*}\left(\tau_{A}\right)$ and, for each $a \in A$, $\Phi(a)$ for $\Phi\left(a, \tau_{A}\right)$.

For each $a \in\left\{p^{*}, p^{*}+1\right\}$, let $H_{a}=\left\{v \in \operatorname{supp}(\nu): \varphi\left(v, \tau_{A}\right)=\{a\}\right\}$ and, for each $a \in A_{0}\left(\tau_{A}\right)$, let $H_{a}=\{v \in \operatorname{supp}(\nu):(v, a) \in \operatorname{supp}(\tau)\}$. As in the proof of part (a) of Theorem 6 , we obtain an open neighborhood $W$ of $\tau_{A}$ and, for each $a \in A_{0}\left(\tau_{A}\right) \cup$ $\left\{p^{*}, p^{*}+1\right\}$, an open set $O_{a} \subseteq \mathbb{R}_{+}$, with $H_{a} \subseteq O_{a}$ and $O_{a} \cap O_{a^{\prime}}=\emptyset$ for each $a^{\prime} \neq a$, such that $p^{*}(\pi)=p^{*}\left(\tau_{A}\right), \sum_{p \geq p^{*}\left(\tau_{A}\right)} \pi(p)>1 / k$, and

$$
\varphi(v, \pi)= \begin{cases}\{a\} & \text { if } a \in\left\{p^{*}, p^{*}+1\right\} \\ A_{0}\left(\tau_{A}\right) & \text { otherwise }\end{cases}
$$

for each $v \in O_{a}$ and each $\pi \in W$. Define $h: \bigcup_{a \in A_{0}\left(\tau_{A}\right) \cup\left\{p^{*}, p^{*}+1\right\}} O_{a} \rightarrow A$ by setting $h(u)=a$ for each $u \in O_{a}$; then $h$ is continuous.

The remainder of the argument is analogous to the proof of of Theorem 2, using Lemma 12 instead of Lemma 7.

\section{References}

Anderson, R. (1991): "Nonstandard Analysis with Applications to Economics," in Handbook of Mathematical Economics, vol. 4. North-Holland, Amsterdam.

Aumann, R. (1964): "Markets with a Continuum of Traders," Econometrica, 32, 39-50.

Azevedo, E., And E. Budish (2019): "Strategy-proofness in the Large," Review of Economic Studies, 86, 81-116. 
Brown, D., And A. Robinson (1972): "A Limit Theorem on the Cores of Large Standard Exchange Economies," Proceedings of the National Academy of Sciences, $69,1258-1260$.

Carmona, G. (2004): "On the Purification of Nash Equilibria of Large Games," Economics Letters, 85, 215-219.

Carmona, G., and K. Podczeck (2009): "On the Existence of Pure Strategy Nash Equilibria in Large Games," Journal of Economic Theory, 144, 1300-1319.

- (2012a): "Approximation and Characterization of Nash Equilibria of Large Games," University of Surrey and Universität Wien.

(2012b): "Ex-Post Stability of Bayes-Nash Equilibria of Large Games," Games and Economic Behavior, 74, 418-430.

(2014): "Existence of Nash Equilibrium in Games with a Measure Space of Players and Discontinuous Payoff Functions," Journal of Economic Theory, 152, $130-178$.

(2019): "Strict Pure Strategy Nash Equilibria in Large Finite-Player Games," University of Surrey and Universität Wien.

Castaing, C., and M. Valadier (1977): Convex Analysis and Measurable Multifunctions, vol. 580 of Lect. Notes Math. Springer-Verlag, New York.

Deb, J., And E. Kalai (2015): "Stability in Large Bayesian Games with Heterogeneous Players," Journal of Economic Theory, 157, 1041-1055.

Georgescu-Roegen, N. (1979): "Methods in Economic Science," Journal of Economic Issues, 13, 317-328.

Gradwohl, R., And O. Reingold (2010): "Partial Exposure in Large Games," Games and Economic Behavior, 68, 602-613.

Green, E. (1984): "Continuum and Finite-Player Noncooperative Models of Competition," Econometrica, 52, 975-993. 
Hildenbrand, W. (1970): "On Economies with Many Agents," Journal of Economic Theory, 2, 161-188.

(1974): Core and Equilibria of a Large Economy. Princeton University Press, Princeton.

Housman, D. (1988): "Infinite Player Noncooperative Games and the Continuity of the Nash Equilibrium Correspondence," Mathematics of Operations Research, 13, $488-496$.

KalaI, E. (2004): "Large Robust Games," Econometrica, 72, 1631-1665.

Kalai, E., And E. Shmaya (2018): "Large Strategic Dynamic Interactions," Northwestern University.

Khan, M. A. (2014): "Representation, Language, and Theory: Georgescu-Roegen on Methods in Economic Science," Journal of Economic Issues, 48, 49-87.

Khan, M. A., K. Rath, Y. Sun, and H. Yu (2013): "Large Games with a BioSocial Typology," Journal of Economic Theory, 148, 1122-1149.

Khan, M. A., K. Rath, H. Yu, and Y. Zhang (2017): "On the Equivalence of Large Individualized and Distributionalized Games," Theoretical Economics, 12, $533-554$.

Khan, M. A., And Y. Sun (1996): "Nonatomic Games on Loeb Spaces," Proceedings of the National Academy of Sciences, 93, 15518-15521.

_ (1999): "Non-Cooperative Games on Hyperfinite Loeb Spaces," Journal of Mathematical Economics, 31, 455-492.

(2002): "Non-Cooperative Games with Many Players," in Handbook of Game Theory, Volume 3, ed. by R. Aumann, and S. Hart. Elsevier, Holland.

Koopmans, T. (1974): "Is the Theory of Competitive Equilibrium With It?," American Economic Review, 64, 325-329. 
Loeb, P., And M. WolfF (eds.) (2015): Nonstandard Analysis for the Working Mathematician. Springer, Dordrecht, second edn.

Mandelkern, M. (1990): "On the Uniform Continuity of Tietze Extensions," Archiv der Mathematik, 55, 387-388.

Mas-Colell, A. (1984): "On a Theorem by Schmeidler," Journal of Mathematical Economics, 13, 201-206.

(1985): The Theory of General Economic Equilibrium: A Differentiable Approach. Cambridge University Press, Cambridge.

QiaO, L., And H. Yu (2014): "On the Space of Players in Idealized Limit Games," Journal of Economic Theory, 153, 177-190.

RAshid, S. (1983): "Equilibrium Points of Non-atomic Games: Asymptotic Results," Economics Letters, 12, 7-10.

_ (1987): Economies with Many Agents: An Approach using Nonstandard Analysis. Johns Hopkins Press, Baltimore.

— (1992): "A Direct Proof of Purification for Schmeidler's Theorem," Applied Mathematics Letters, 5, 23-24.

Schmeidler, D. (1973): "Equilibrium Points of Nonatomic Games," Journal of Statistical Physics, 4, 295-300.

YAng, J. (2017): "A Link between Semi-anonymous Sequential Games and their Large Finite Counterparts," International Journal of Game Theory, 46, 383-433. 